\title{
Approche stochastique du modèle RCTD pour la mesure de l'activité en Cherenkov et par scintillation liquide
}

\author{
Stochastic approach of the TDCR model \\ for activity measurement using Cherenkov \\ and liquid scintillation
}

\author{
Christophe BOBIN, Cheick THIAM, Bruno CHAUVENET et Jacques BOUCHARD
}

CEA, List, Laboratoire national Henri Becquerel (LNE-LNHB), 91191 Gif-sur-Yvette Cedex, France, christophe.bobin@cea.fr.

\section{Résumé}

En métrologie de l'activité, la méthode RCTD (Rapport des Coöncidences Triples à Doubles) est une technique primaire fondée sur la scintillation liquide, utilisant une instrumentation spécifique à trois photomultiplicateurs. Le modèle RCTD statistique de l'émission lumineuse permettant le calcul des rendements de détection des coïncidences est étudié dans le cas de dépôts de basses énergies dans le liquide scintillant. Pour cela, l'indépendance stochastique entre les photomultiplicateurs est introduite comme condition préalable à l'écriture des relations classiques du modèle statistique. Deux phénomènes pouvant conduire à la non-validité de cette condition sont décrits. Mise en évidence expérimentalement avec la mesure d'activité du tritium $\left({ }^{3} \mathrm{H}\right)$, la dépendance stochastique de type temporel se manifeste par une perte systématique de coïncidences lorsque le temps de résolution est trop court comparé à la distribution temporelle des photons de scintillation. La dépendance stochastique de type géométrique est également étudiée en raison de la sensibilité du comptage des coïncidences selon le point d'origine de l'émission lumineuse dans le volume du liquide scintillant. Cette étude a été possible grâce au développement d'une nouvelle modélisation avec le code Monte-Carlo Geant4 intégrant la simulation de la propagation des photons optiques jusqu'à la production des photoélectrons dans le détecteur. Dans les deux cas de dépendance stochastique, l'influence sur le calcul des rendements de détection des coïncidences et par suite sur la détermination de l'activité est exposée. Le nouveau modèle RCTD-Geant4 intègre aussi l'émission de photons Cherenkov; il a permis la confirmation de l'émission de lumière «parasite » sous forme de photons Cherenkov suite à la diffusion Compton dans les faces d'entrée des photomultiplicateurs. Une nouvelle technique primaire RCTD-Cherenkov a également été développée grâce au modèle RCTD-Geant4; elle a été notamment appliquée à la mesure d'activité du carbone-11 (radiopharmaceutique à durée de vie courte, $T_{1 / 2}$ de l'ordre de $20 \mathrm{~min}$ ).

MOTS CLÉS : MÉTROLOGIE DE L'ACTIVITÉ, MÉTHODE RCTD, SCINTILLATION LIQUIDE, EFFET CHERENKOV, SIMULATION MONTE-CARLO, CODE GÉANT4.

\begin{abstract}
In the field of radionuclide metrology, the TDCR method is a primary technique based on liquid scintillation using a threephotomultiplier instrumentation. The application of the TDCR model classically implemented in laboratories is investigated in the case of low-energy deposition in the liquid scintillator. For that purpose, the condition of stochastic independence between photomultipliers is introduced in order to express the classical probabilistic relations used for the detection efficiencies of coincidences. Experimentally observed in the case of ${ }^{3} \mathrm{H}$ standardization, the time dependence arises when the coincidence resolving time is shorter than the time distribution of scintillation photons. The geometric dependence is also investigated because of the sensitivity of coincidence counting with the position of light emission inside the volume of the liquid scintillator. For that purpose, a new TDCR modeling using the Monte Carlo code Geant4 has been implemented in order to simulate the propagation of photons from their creation in the optical chamber to the production of photoelectrons in liquid scintillation counter. In both cases of stochastic dependence, the influence on the calculation of detection efficiencies and thus on the activity determination is presented. The new TDCR-Geant4 model was also developed to take into account Cherenkov emission; it has been applied to confirm the emission of Cherenkov photons subsequently created by Compton scattering in the photomultiplier windows. Using the TDCR-Geant4 model, a new primary technique is also presented by implementing the TDCR method using the Cherenkov emission. This new technique has been applied to the standardization of the short-lived radiopharmaceutical ${ }^{11} \mathrm{C}\left(T_{1 / 2}\right.$ about $\left.20 \mathrm{~min}\right)$.
\end{abstract}

KEY WORDS: RADIONUCLIDE METROLOGY, TDCR METHOD, LIQUID SCINTILLATION, CHERENKOV EFFECT, MONTE CARLO SIMULATION, GEANT4 CODE.

\section{Introduction}

Largement employée dans les laboratoires nationaux de métrologie de la radioactivité, la méthode RCTD 
(Rapport des Coïncidences Triples à Doubles) est une technique primaire de mesure d'activité fondée sur la scintillation liquide [1]. Son application repose sur un système de détection spécifique composé de trois photomultiplicateurs disposés autour d'une chambre optique (fig. 1). Celle-ci est conçue pour recevoir un flacon contenant un mélange constitué d'un liquide scintillant et de solution radioactive. N'utilisant pas d'étalon radioactif, cette méthode nécessite le calcul du rendement de détection à partir d'un modèle statistique de l'émission lumineuse conduisant aux comptages de photoélectrons dans les photomultiplicateurs. En pratique, la mesure d'activité est effectuée à partir du comptage des coïncidences doubles et triples entre les photomultiplicateurs. Connaissant le schéma de désintégration du radionucléide, le rendement de détection des coïncidences doubles est calculé à partir du rapport $R C T D$ expérimental.

Parmi les études et développements réalisés récemment sur la méthode RCTD, on distinguera deux thématiques. D'une part, l'utilisation d'une nouvelle instrumentation électronique [2] a été l'occasion d'entreprendre l'étude systématique de l'influence de la durée du temps de résolution sur le comptage des coïncidences pour divers radionucléides. D'autre part, une nouvelle modélisation de l'émission lumineuse avec le code Monte-Carlo Geant4 [3] permet d'aller au-delà du modèle RCTD statistique : elle simule la propagation des photons optiques à l'intérieur de la chambre optique, de leur création dans le flacon jusqu'à la production de photoélectrons dans les photomultiplicateurs [4]. L'intérêt de cette approche stochastique est la prise en compte de l'ensemble des processus associés à l'optique géométrique tels que la réfraction et la réflexion aux interfaces de la cavité optique. Ce nouveau modèle RCTD-Geant4 intègre également l'émission de photons par effet Cherenkov.

Les différentes étapes de la construction du modèle RCTD statistique sont exposées en partant de la condition d'indépendance stochastique entre les photomultiplicateurs [5]. Bien que ce prérequis ne soit pas mentionné dans les travaux de recherche publiés, il est démontré que celui-ci est indispensable à l'écriture des relations classiques des probabilités de détection entre les photomultiplicateurs. Pour cette nouvelle approche, le formalisme des probabilités conditionnelles est utilisé ; il sera particulièrement utile à la compréhension des biais constatés pour la mesure d'activité d'émetteurs de rayonnements de basse énergie (inférieure à $20 \mathrm{keV}$ ). Deux phénomènes pouvant avoir une incidence sur l'indépendance stochastique entre les photomultiplicateurs sont étudiés : l'un d'origine temporelle lorsque la variation des taux de coïncidences est sensible à la valeur du temps de résolution, l'autre de nature géométrique due à l'origine de l'émission lumineuse dans le volume du liquide scintillant (à l'intérieur du flacon). L'impact de la dépendance stochastique de type temporel est présenté pour la mesure d'activité du tritium $\left({ }^{3} \mathrm{H}\right)$; celle de type géométrique, dans le cas de la mesure du chrome-51 $\left({ }^{51} \mathrm{Cr}\right)$.

Il sera aussi question d'un autre phénomène non considéré dans le modèle RCTD statistique. Il s'agit de

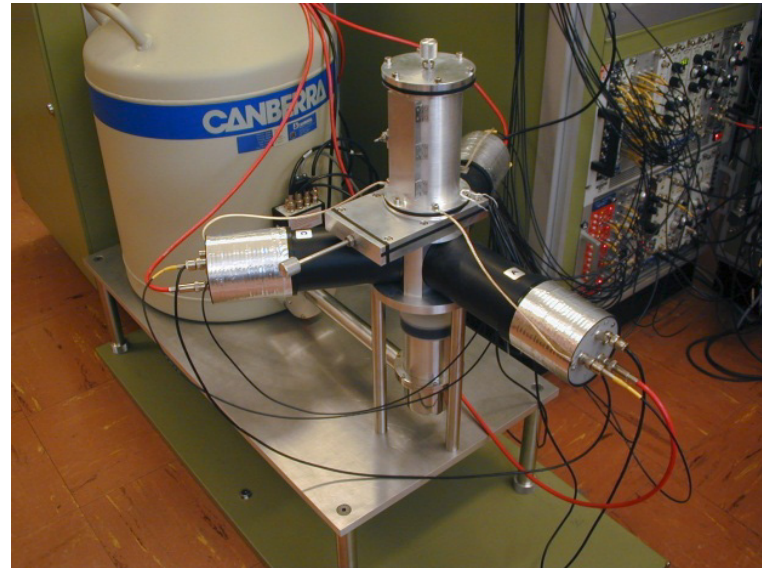

Fig. 1. - Le détecteur à trois photomultiplicateurs est représenté au premier plan. Ces trois photorécepteurs entourent un flacon de liquide scintillant placé dans une cavité optique. L'activité d'un radionucléide est déterminée à partir des taux de coïncidences doubles et triples entre les photomultiplicateurs.

l'émission de photons Cherenkov dans les faces d'entrée des photomultiplicateurs suite à la diffusion Compton de photons $\gamma$ émis par la solution radioactive contenue dans le flacon. D'abord mis en évidence expérimentalement, cet effet a été confirmé grâce au nouveau modèle RCTD-Geant4 [4]. L'influence de cette lumière «parasite » est présentée dans le cas de la mesure d'activité du manganèse-54 $\left({ }^{54} \mathrm{Mn}\right)$.

Enfin l'utilisation du nouveau modèle RCTD-Geant4 a permis le développement d'une nouvelle technique de mesure basée exclusivement sur l'effet Cherenkov. D'abord validée avec la mesure d'activité de l'yttrium$90\left({ }^{90} \mathrm{Y}\right)$ [6], cette technique RCTD-Cherenkov est directement appliquée en solution aqueuse (ce qui facilite la préparation des sources radioactives). La mesure d'activité du carbone-11 (période radioactive $T_{1 / 2}$ de l'ordre de $20 \mathrm{~min}$ ) par la technique RCTD-Cherenkov est détaillée [7].

\section{Le modèle RCTD statistique}

\subsection{Relations générales des probabilités de détection entre les photomultiplicateurs}

Dans cette partie, les relations de probabilités du modèle RCTD statistique sont abordées sans aucune hypothèse particulière sur le système de détection à trois photomultiplicateurs.

Si l'on considère l'énergie $E$ déposée dans le liquide scintillant suite à la désintégration d'un radionucléide, l'évènement correspondant à la « détection dans la voie de comptage $i$ d'une désintégration » est noté $x_{i}$ et la probabilité de détection correspondante $P_{E}\left(x_{i}\right)$. La probabilité mutuelle $P_{E}\left(x_{1} \cap x_{2}\right)$ définit la détection dans les deux voies 1 et 2 d'une même désintégration, désignée comme une coïncidence double. Lorsqu' aucune hypothèse n'est avancée sur l'indépendance stochastique entre les voies, la probabilité mutuelle s'exprime classiquement en fonction de la probabilité conditionnelle $P_{E}\left(x_{2} \mid x_{1}\right)$ d'avoir une 
détection dans la voie 2 sachant qu'une détection se produit dans la voie 1 :

$$
P_{E}\left(x_{1} \cap x_{2}\right)=P_{E}\left(x_{1}\right) \cdot P_{E}\left(x_{2} \mid x_{1}\right) .
$$

De façon similaire, la probabilité mutuelle $P_{E}\left(x_{1} \cap x_{2} \cap x_{3}\right)$ définit la détection dans les trois voies 1,2 et 3 d'une même désintégration, désignée comme une coïncidence triple; elle est fonction de la probabilité conditionnelle $P_{E}\left(x_{3} \mid x_{1} \cap x_{2}\right)$ d'avoir une détection dans la voie 3 sachant qu'une coïncidence double se produit dans les voies 1 et 2 ,

$$
P_{E}\left(x_{1} \cap x_{2} \cap x_{3}\right)=P_{E}\left(x_{1}\right) \cdot P_{E}\left(x_{2} \mid x_{1}\right) \cdot P_{E}\left(x_{3} \mid x_{1} \cap x_{2}\right) .
$$

Par suite, on obtient les relations de probabilités correspondant aux rendements de détection $R_{D}(E)$ pour les coïncidences doubles et $R_{T}(E)$ pour les coïncidences triples :

$$
\begin{aligned}
R_{D}(E)= & P_{E}\left(x_{1} \cap x_{2}\right)+P_{E}\left(x_{2} \cap x_{3}\right)+P_{E}\left(x_{1} \cap x_{3}\right) \\
& -2 P_{E}\left(x_{1} \cap x_{2} \cap x_{3}\right) \\
R_{T}(E)= & P_{E}\left(x_{1}\right) \cdot P_{E}\left(x_{2} \mid x_{1}\right) \cdot P_{E}\left(x_{3} \mid x_{1} \cap x_{2}\right) \\
= & P_{E}\left(x_{1} \cap x_{2} \cap x_{3}\right) .
\end{aligned}
$$

\subsection{Relations classiques}

Les relations définies précédemment grâce au formalisme des probabilités conditionnelles ne sont pas mentionnées dans les articles spécialisés. Dans la construction du modèle RCTD statistique « classique », les relations ne font apparaître que les probabilités individuelles de détection dans les trois voies de détection. Cette écriture est en réalité conditionnée par l'hypothèse implicite de l'indépendance stochastique entre les trois voies de détection. En effet, ce n'est que lorsque cette condition s'applique, que les expressions (1), (2), (3) et (4) se simplifient et peuvent s'écrire à partir des probabilités individuelles de détection.

On a dans ce cas :

$$
\begin{aligned}
P_{E}\left(x_{2} \mid x_{1}\right) & =P_{E}\left(x_{2}\right), & P_{E}\left(x_{3} \mid x_{1}\right) & =P_{E}\left(x_{3}\right), \\
P_{E}\left(x_{2} \mid x_{3}\right) & =P_{E}\left(x_{2}\right) \quad \text { et } & P_{E}\left(x_{3} \mid x_{1} \cap x_{2}\right) & =P_{E}\left(x_{3}\right) .
\end{aligned}
$$

Ainsi, les rendements de détection des coïncidences doubles et triples donnés en (3) et (4) se simplifient :

$$
\begin{aligned}
R_{D}(E)= & P_{E}\left(x_{1}\right) \cdot P_{E}\left(x_{2}\right)+P_{E}\left(x_{2}\right) \cdot P_{E}\left(x_{3}\right) \\
& +P_{E}\left(x_{1}\right) \cdot P_{E}\left(x_{3}\right) \\
& -2 P_{E}\left(x_{1}\right) \cdot P_{E}\left(x_{2}\right) \cdot P_{E}\left(x_{3}\right) \\
R_{T}(E)= & P_{E}\left(x_{1}\right) \cdot P_{E}\left(x_{2}\right) \cdot P_{E}\left(x_{3}\right) .
\end{aligned}
$$

Le problème de l'asymétrie entre les trois voies de comptage a été traité par Broda et coll. [1]. Dans la suite, le modèle RCTD statistique est développé pour des photomultiplicateurs identiques. Cette configuration conduit aux égalités suivantes :

$$
P_{E}\left(x_{1}\right)=P_{E}\left(x_{2}\right)=P_{E}\left(x_{3}\right)=P_{E}(x) .
$$

Les rendements de détection des coïncidences doubles et triples s'expriment alors en fonction de la probabilité individuelle $P_{E}(x)$ :

$$
\begin{aligned}
& R_{D}(E)=3 P_{E}(x)^{2}-2 P_{E}(x)^{3}, \\
& R_{T}(E)=P_{E}(x)^{3} .
\end{aligned}
$$

Dans le modèle RCTD statistique «classique », le nombre de photons émis suite à un dépôt d'énergie $E$, est décrit par une distribution de Poisson dont l'espérance mathématique est désignée par $m(E)$. L'expression $P_{E}(x)$ est définie comme la probabilité de compter au moins un photoélectron suite à une désintégration; elle s'écrit comme le complément de la probabilité de Poisson de non-détection de photoélectrons où $v$ est le rendement quantique de la photocathode d'un photomultiplicateur :

$$
P_{E}(x)=1-\exp (-m(E) \cdot v / 3)
$$

On remarque que la répartition des photons est supposée équiprobable entre les trois photomultiplicateurs.

Par ailleurs, l'émission de lumière dans le liquide scintillant n'est pas linéaire avec le dépôt d'énergie (en particulier pour des électrons de basse énergie). L'atténuation lumineuse due au quenching d'ionisation dépend du transfert linéique d'énergie du rayonnement ionisant dans le scintillateur. Ce phénomène est décrit dans le modèle RCTD en utilisant la formule semi-empirique de Birks [8] :

$$
m(E)=A \int_{0}^{E} \frac{\mathrm{d} E}{1+k B \frac{\mathrm{d} E}{\mathrm{~d} x}},
$$

où $A$ est le rendement de scintillation par unité d'énergie déposée; $\mathrm{d} E / \mathrm{d} x$ est le transfert linéique d'énergie du rayonnement ionisant et $k B$ un facteur caractéristique du scintillateur.

Au final, les moyennes arithmétiques des grandeurs $R C T D$ et des rendements de détection des coïncidences doubles $R_{D}$ et triples $R_{T}$ sont obtenues en intégrant les expressions (9) et (10) selon les fréquences $S(E)$ associées à la distribution des énergies déposées dans le scintillateur. Celles-ci dépendent du schéma de désintégration du radionucléide. On obtient :

$$
\begin{aligned}
R_{T} & =\int_{S} S(E) \cdot P_{E}(x)^{3} \cdot \mathrm{d} E, \\
R_{D} & =\int_{S} S(E) \cdot\left(3 P_{E}(x)^{2}-2 P_{E}(x)^{3}\right) \mathrm{d} E, \\
R C T D & =R_{T} / R_{D} .
\end{aligned}
$$

En pratique, la valeur $R C T D$ expérimentale est obtenue à partir des comptages des coïncidences triples et doubles. Le calcul du rendement de détection des coïncidences doubles est implémenté par optimisation en ajustant le paramètre libre $A v$ pour obtenir l'égalité entre les valeurs $R C T D$ expérimentale et calculée. 


\subsection{Relations spécifiques du modèle RCTD statistique pour un dépôt monoénergétique $E$}

Pour les études décrites dans la suite de l'article concernant l'indépendance stochastique entre les photomultiplicateurs, une relation simple entre le rendement de détection des coïncidences doubles et la valeur RCTD est déduite des expressions (9) et (10). Dans le cas particulier d'un dépôt monoénergétique $E$, les rendements de détection $R_{D}$ et $R_{T}$ sont respectivement égaux à $R_{D}(E)$ et $R_{T}(E) ; P(x)$ est égal à $P_{E}(x)$. On a :

$$
\frac{R_{T}}{R_{D}}=R C T D=\frac{P(x)}{(3-2 P(x))} .
$$

Ainsi, il est possible d'écrire une relation simple entre $R_{D}$ et $R C T D$ :

$$
R_{D}=27 \frac{R C T D^{2}}{(1+2 R C T D)^{3}} .
$$

\subsection{Commentaires}

La condition d'indépendance stochastique entre les photomultiplicateurs comme préalable à l'application du modèle RCTD statistique «classique » n'a jamais été mentionnée explicitement dans les articles spécialisés. A contrario, ce prérequis avait été pris en considération dès ses débuts pour la méthode des coïncidences $4 \pi \beta-\gamma$ dont l'application repose sur l'hypothèse d'indépendance stochastique entre les voies $\beta$ et $\gamma$ [9]. Lorsque cette condition est vérifiée, le taux de comptage des coïncidences peut s'exprimer comme le produit de l'activité et des rendements de détection des voies $\beta$ et $\gamma$.

Lorsque l'indépendance stochastique entre les photomultiplicateurs ne s'applique pas, l'équivalence des expressions (3) et (6) pour les coïncidences doubles, et (4) et (7) pour les coïncidences triples, n'existe plus. Cette situation conduit à un problème de validité du modèle RCTD statistique «classique » qui, en s'écartant significativement du comportement réel du comptage des coïncidences, peut engendrer un biais dans la détermination de l'activité.

L'un des objectifs des études présentées dans cet article est d'identifier les facteurs possibles de dépendance stochastique entre les photomultiplicateurs. D'abord, l'hypothèse d'une dépendance stochastique de type temporel est étudiée pour comprendre l'influence du temps de résolution sur la mesure de l'activité de ${ }^{3} \mathrm{H}(\S 4)$ et plus généralement des émetteurs de basses énergies. Cette étude est ensuite étendue à la dépendance stochastique de type géométrique ( $\$ 5$ ). Elle a été rendue possible grâce au développement d'un nouveau modèle RCTD basé sur le code de simulation Geant4. À la différence du modèle statistique «classique », il permet de suivre les différents phénomènes optiques (réfraction et réflexion) se produisant dans le système de détection depuis la création et la propagation des photons optiques dans la cavité optique jusqu'à la création des photoélectrons dans les photomultiplicateurs. L'étude porte sur l'influence de la position d'origine de l'émission lumineuse dans le flacon contenant le liquide scintillant sur la dépendance stochastique entre les photomultiplicateurs.

\section{3. Étude expérimentale de l'influence du temps de résolution sur le taux de coïncidences}

\subsection{Instrumentation}

Les différents résultats présentés dans cet article ont été obtenus avec un système de détection composé de trois photomultiplicateurs XP2020Q (Photonis) disposés symétriquement autour d'une chambre optique. Constitué d'une face d'entrée en silice fondue, le domaine spectral de sensibilité du photomultiplicateur est compris entre $160 \mathrm{~nm}$ et $650 \mathrm{~nm}$. Développé pour la scintillation liquide, ce détecteur est aussi sensible aux photons Cherenkov dont l'émission s'étend jusqu'au domaine ultraviolet du spectre. La cavité réflectrice en Téflon ${ }^{\circledR}$ dans laquelle est placée le flacon est de forme sphérique.

Dans les trois voies de comptage, les signaux analogiques délivrés par les photomultiplicateurs sont d'abord amplifiés pour ensuite alimenter un module de discrimination rapide à fraction constante d'amplitude (FCA). Ce dernier délivre des signaux logiques dont le seuil de déclenchement est réglé pour être sensible au photoélectron unique généré par l'émission thermoélectronique de la photocathode. Un module MAC3 [10] assure ensuite le comptage des coïncidences doubles et triples entre les photomultiplicateurs grâce à un temps de résolution fixé à $40 \mathrm{~ns}$. Une autre fonctionnalité importante de ce module est d'assurer une protection adaptée aux paralysies du système de comptage. Grâce à l'application de la technique du temps actif combinée à l'utilisation des temps morts de type reconductible, ce dispositif évite les excès de comptage dus aux post-impulsions dont l'origine est attribuée aux processus physico-chimiques propres à la scintillation liquide ou encore à l'ionisation du gaz résiduel dans les photomultiplicateurs. La distribution temporelle de ces post-impulsions peut aller de quelques nanosecondes à plusieurs microsecondes.

\subsection{Développement d'un système électronique de comptage à logiques programmables}

Pour répondre aux difficultés d'assurer la pérennité des installations de mesure actuelles, il a été décidé d'opérer une transition vers la technologie numérique à base de circuits programmables tels que FPGA (Field Programmable Gates Array) ou DSP (Digital Signal Processor). Depuis quelques années, une plateforme généraliste pouvant être adaptée à différentes méthodes primaires de mesure d'activité est en cours de développement. Elle doit répondre aux objectifs principaux suivants :

- possibilité de connecter les détecteurs usuels en métrologie de la radioactivité;

- implémentation en temps réel du comptage, des temps morts de type reconductible et de la technique du temps actif ; 
- possibilité d'associer deux systèmes de détection (par exemple, compteur à scintillation liquide et détecteur à semi-conducteur de type HPGe) pour l'application de la méthode des coïncidences $4 \pi \beta-\gamma$ [9].

Pour répondre à ces critères, une carte de développement produite par la société Altera a été choisie. Le composant principal est un circuit FPGA Stratix ${ }^{\circledR}$ III comprenant environ 140000 éléments logiques. Sa programmation avec le code VHDL a été sous-traitée à une société spécialisée. La carte numérique possède également deux connecteurs de type HSMC (High Speed Mezzanine Card) permettant l'ajout de cartes pour des fonctionnalités supplémentaires. Un de ces connecteurs est utilisé pour coupler la plateforme numérique à une carte comprenant deux convertisseurs analogiques-numériques d'une profondeur de codage de 14 bits et d'une fréquence d'échantillonnage maximale de $150 \mathrm{MHz}$. La carte numérique dispose également d'autres éléments, comme de la mémoire sous forme d'une barrette de 1 Go DDR2 SDRAM; celle-ci peut être utilisée pour d'autres applications telles que le stockage de données temporelles associées aux impulsions délivrées par les photomultiplicateurs pour un traitement hors ligne de l'information.

La faisabilité d'un traitement en temps réel des comptages et des temps morts a été validée avec le système de détection à scintillation liquide pour appliquer la méthode RCTD. Les fonctionnalités programmées pour le comptage des coïncidences sont proches de celles déjà existantes dans le module MAC3. De nouvelles fonctionnalités ont été ajoutées : un temps de résolution réglable est disponible pour le comptage des coïncidences et des mesures temporelles sont effectuées en temps réel comme le ferait un convertisseur temps-numérique. Les différentes opérations implémentées par des modules séparés dans une instrumentation classique sont directement programmées au niveau du circuit FPGA. Le lien avec un ordinateur d'acquisition est assuré par une connexion Ethernet.

Le comptage en temps réel des coïncidences avec le système numérique a été validé grâce à la mesure de l'activité de ${ }^{241} \mathrm{Am}$ dans le scintillateur commercial Ultima Gold. Pour le calcul de l'activité de cet émetteur $\alpha$, le rendement de détection peut être pris égal à l'unité. Ces premières mesures indiquent que la comparaison avec l'instrumentation classique constituée du module MAC3 n'a conduit à aucune différence significative même lorsque le temps de résolution est étendu à $120 \mathrm{~ns}$ avec le système numérique. Un temps de résolution de $15 \mathrm{~ns}$ serait suffisant pour effectuer une mesure correcte sans perte de coïncidences.

\subsection{Influence du temps de résolution dans la mesure de l'activité de ${ }^{3} \mathbf{H}$}

La comparaison entre les deux systèmes de comptage a été étendue à la mesure de l'activité de ${ }^{3} \mathrm{H}$ dans le scintillateur Ultima Gold. Ce radionucléide se désintègre par émission $\beta^{-}$avec une énergie maximale de $18,6 \mathrm{keV}$ environ. Comme le montre la figure 2 , on observe une

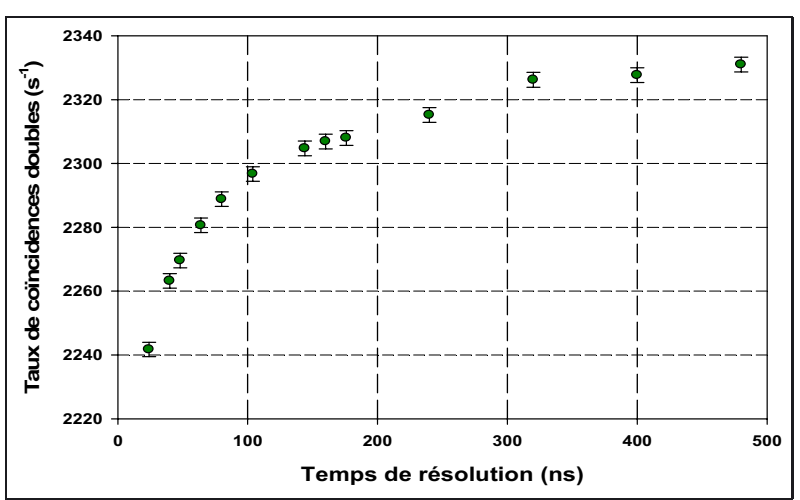

Fig. 2. - Évolution du taux de comptage des coïncidences doubles en fonction du temps de résolution pour la mesure de l'activité de ${ }^{3} \mathrm{H}$ dans le scintillateur Ultima Gold. L'écart relatif entre 40 ns et 250 ns est de $2,5 \%$.

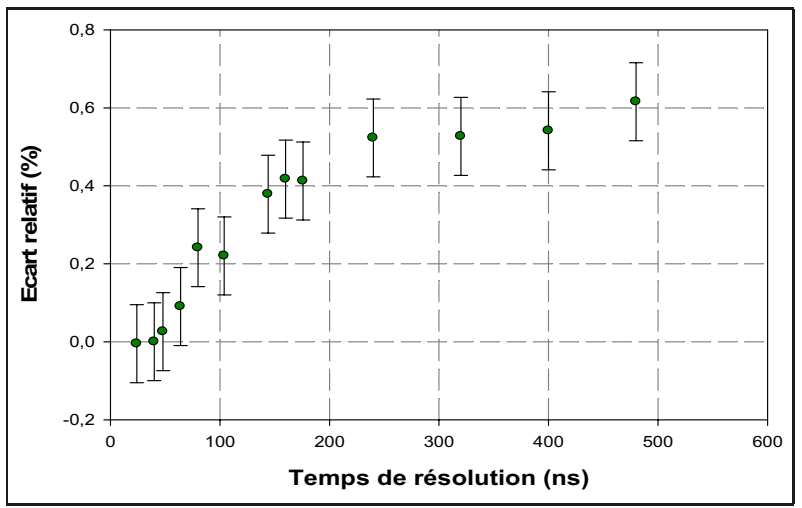

Fig. 3. - Écart relatif de l'activité de ${ }^{3} \mathrm{H}$ calculée avec le modèle RCTD statistique à partir des taux de coïncidences doubles donnés en figure 2. L'activité déterminée avec un temps de résolution égal à $40 \mathrm{~ns}$ est prise comme référence (correspondant à la valeur donnée par l'instrumentation classique avec le module MAC3).

dépendance significative du taux de comptage des coïncidences doubles avec la valeur du temps de résolution. Des résultats montrant le même phénomène ont été publiés par le laboratoire national australien (ANSTO) [11]. La variation est de l'ordre de $+2,5 \%$ entre 40 ns (valeur fixée dans le module MAC3) et 250 ns de temps de résolution; une augmentation de la valeur RCTD de 0,53 à 0,55 est également observée.

Dans le but de connaître l'incidence de ces résultats sur l'estimation de l'activité de ${ }^{3} \mathrm{H}$, le modèle RCTD statistique, décrit dans la partie 2.2, a été appliqué en utilisant un facteur $k B$ égal à $0,01 \mathrm{~cm} \cdot \mathrm{MeV}^{-1}$. Les valeurs d'activité en fonction du temps de résolution sont données en figure 3 : une augmentation de l'ordre de 0,5\% demeure entre $40 \mathrm{~ns}$ et $250 \mathrm{~ns}$; un plateau apparaît à partir de cette dernière valeur. L'hypothèse avancée lors de la publication de ces résultats [2] était la suivante : en raison du petit nombre de photons émis suite au transfert d'une faible quantité d'énergie au liquide scintillant, le comptage des coïncidences doubles et triples devient sensible à la distribution temporelle des photons entre 
les photomultiplicateurs à cause de la décroissance de la fluorescence.

\subsection{Mesures complémentaires}

Pour étayer l'hypothèse émise sur la base des résultats obtenus avec ${ }^{241} \mathrm{Am}$ et ${ }^{3} \mathrm{H}$, l'influence du temps de résolution sur le comptage des coïncidences a été étudiée pour d'autres radionucléides. Dans le cas de positons de haute énergie émis par ${ }^{18} \mathrm{~F}\left(E_{\beta}^{+}\right.$max. de l'ordre de $633 \mathrm{keV}$ ) dans Ultima Gold, les conclusions sont semblables à celles déduites des résultats de ${ }^{241} \mathrm{Am}$ : on n'observe pas la variation du comptage des coïncidences doubles avec le temps de résolution constatée pour ${ }^{3} \mathrm{H}$.

Les mesures réalisées avec ${ }^{99 \mathrm{~m}} \mathrm{Tc}$ dans Ultima Gold sont particulièrement intéressantes. La figure 4 montre l'évolution du taux de comptages des coïncidences doubles et triples en fonction du temps de résolution sous forme de pourcentages en prenant pour référence les taux obtenus à $40 \mathrm{~ns}$. De façon contre-intuitive, on constate que les écarts observés pour les coïncidences triples sont inférieurs à ceux obtenus avec les coïncidences doubles. Ce résultat inattendu est la conséquence du schéma de désintégration de ${ }^{99 \mathrm{~m}} \mathrm{Tc}$. En effet, ce radionucléide se désintègre principalement selon une cascade de deux transitions $\gamma$ [12] : la première d'une énergie de l'ordre de $2 \mathrm{keV}$ est très convertie; la seconde d'environ $140 \mathrm{keV}$ est peu convertie. Les électrons de conversion de la première transition interagissent avec une probabilité proche de 1 dans le scintillateur tout en générant un faible nombre de photons, conduisant majoritairement à la détection de coïncidences doubles. A contrario, les photons $\gamma$ de la seconde transition interagissent dans le scintillateur avec une probabilité beaucoup plus faible, mais en produisant un nombre de photons suffisamment élevé pour générer en majorité des coïncidences triples. Ainsi les coïncidences doubles principalement dues aux électrons de $2 \mathrm{keV}$ suivent une évolution en fonction du temps de résolution semblable à celle donnée pour ${ }^{3} \mathrm{H}$. Les coïncidences triples principalement dues aux photons $\gamma$ de $140 \mathrm{keV}$ ont une sensibilité moindre à la valeur du temps de résolution.

Comme le montrent les figures 2 et 4 , la réduction du temps de résolution engendre une baisse du rendement de détection du taux de coïncidences. L'équivalence de cet effet avec la variation de la tension de focalisation (ou défocalisation) des photomultiplicateurs a été testée pour deux radionucléides ayant des schémas de désintégration différents : d'une part l'émetteur $\beta^{-},{ }^{3} \mathrm{H}$, donnant un spectre d'énergie continu, d' autre part ${ }^{51} \mathrm{Cr}$ se désintégrant par capture électronique suivant un spectre d'énergie discret (émission d'électrons Auger et de photons X dont les énergies maximales sont comprises entre $4 \mathrm{keV}$ et $6 \mathrm{keV}$ ) [12].

La figure 5 présente, pour ${ }^{3} \mathrm{H}$, les évolutions des taux de coïncidences doubles en fonction de la mesure $R C T D$ obtenus d'une part par défocalisation des photomultiplicateurs (avec un temps de résolution fixé à $240 \mathrm{~ns}$ ), d'autre part par réduction du temps de résolution (entre $240 \mathrm{~ns}$ et $16 \mathrm{~ns}$ ) pour deux tensions de

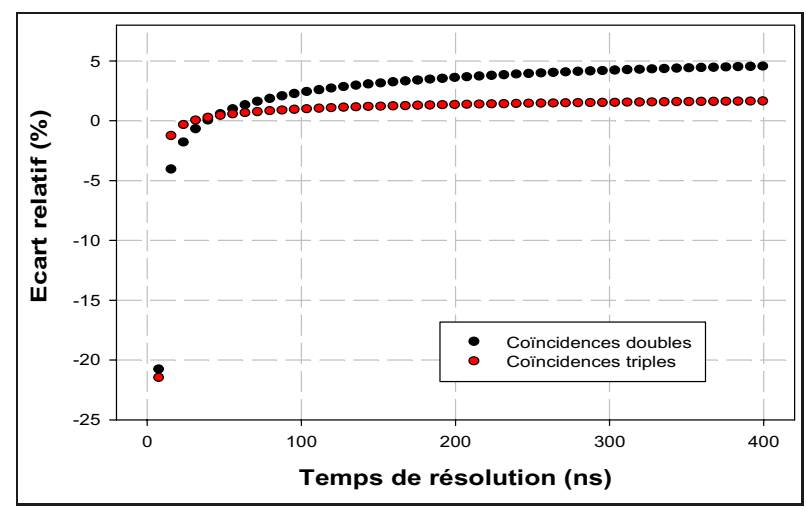

Fig. 4. - Écarts relatifs des taux de comptage des coïncidences en fonction du temps de résolution dans le cas de ${ }^{99 \mathrm{~m}} \mathrm{Tc}$ dans le scintillateur Ultima Gold. Les taux à 40 ns sont pris comme référence.

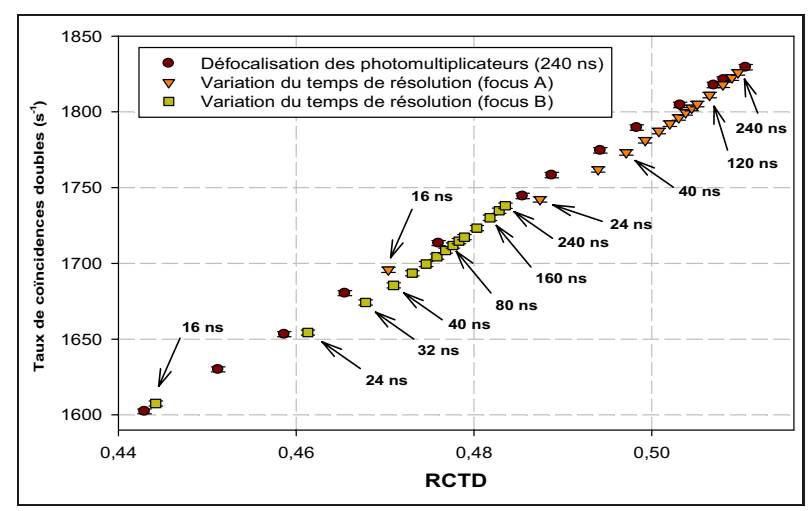

Fig. 5. - Évolutions des taux de coïncidences doubles en fonction de RCTD pour la mesure de l'activité de ${ }^{3} \mathrm{H}$ dans le scintillateur Ultima Gold. La réduction du rendement de détection obtenue par diminution du temps de résolution (selon deux tensions de focalisation $\mathrm{A}$ et $\mathrm{B}$ ) est comparée à celle donnée par défocalisation des photomultiplicateurs (temps de résolution fixé à $240 \mathrm{~ns})$.

focalisation. La non-équivalence observée entre les deux techniques peut être interprétée comme la conséquence de l'influence du temps de résolution sur la relation entre le taux de coïncidences doubles et le rapport $R C T D$ (on rappelle que ces deux valeurs expérimentales sont utilisées pour le calcul de l'activité). Pour la valeur de $R C T D$ obtenue à $40 \mathrm{~ns}$, le taux de coïncidences doubles donné par défocalisation est de $0,5 \%$ supérieur à celui obtenu par réduction du temps de résolution. Cet écart est égal à celui donné dans la partie 3.3 pour le calcul de l'activité de ${ }^{3} \mathrm{H}$ entre $40 \mathrm{~ns}$ et $250 \mathrm{~ns}$. Cette égalité est due à la relation biunivoque entre la valeur RCTD et le rendement de détection des coïncidences doubles.

Par ailleurs, on constate une équivalence entre les deux techniques de variation du rendement de détection des coïncidences pour un temps de résolution de 16 ns. Cependant, comme le montre la figure 6 dans le cas de la mesure de l'activité de ${ }^{51} \mathrm{Cr}$, les résultats tirés de la comparaison avec ${ }^{3} \mathrm{H}$ ne peuvent pas être généralisables. Dans le cas de l'émetteur donnant un spectre d'énergie discret, l'augmentation du taux de coïncidences doubles en 


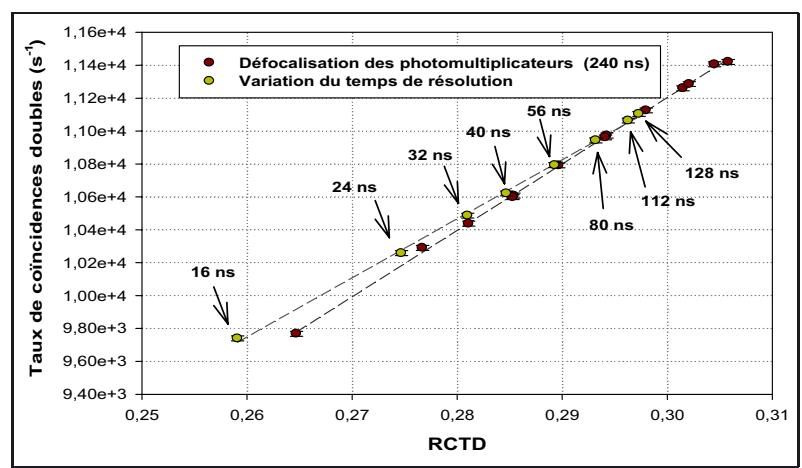

Fig. 6. - Évolutions des taux de coïncidences doubles en fonction de $R C T D$ pour la mesure de l'activité de ${ }^{51} \mathrm{Cr}$ dans le scintillateur Ultima Gold. La réduction du rendement de détection obtenue par diminution du temps de résolution (selon deux tensions de focalisation $\mathrm{A}$ et $\mathrm{B}$ ) est comparée à celle donnée par défocalisation des photomultiplicateurs (temps de résolution fixé à 240 ns).

fonction de la valeur $R C T D$ reste corrélée à l'allongement de la durée du temps de résolution. En revanche, la nonéquivalence n'est pas observable pour les mêmes valeurs du temps de résolution : elle devient significative pour des valeurs inférieures à $50 \mathrm{~ns}$. En outre, contrairement aux mesures de l'activité de ${ }^{3} \mathrm{H}$, la réduction du temps de résolution donne des taux de coïncidences doubles supérieurs à ceux obtenus par défocalisation pour la même valeur de $R C T D$.

\section{Simulation de l'influence de la distribution temporelle des photoélectrons sur la dépendance stochastique entre les photomultiplicateurs}

\subsection{Description}

La simulation de la distribution temporelle des photoélectrons dans les photomultiplicateurs à la suite d'un dépôt d'énergie dans le scintillateur a pour objectif l'étude de l'influence du temps de résolution sur le comptage des coïncidences, sur la dépendance stochastique entre les photomultiplicateurs, et sur le calcul de l'activité. Les calculs ont été effectués sur la base du modèle RCTD monoénergétique décrit dans la partie 2.3. La variabilité des séquences temporelles des photoélectrons est prise en compte grâce à un échantillonnage exhaustif obtenu à partir de la simulation de dépôts monoénergétiques successifs dans le liquide scintillant. De ces séquences temporelles on tire l'instant correspondant au premier photoélectron détecté dans chaque photomultiplicateur. Il est ensuite possible de calculer les coïncidences triples et doubles en fonction du temps de résolution. Considérés comme des post-impulsions, les photoélectrons subséquents ne sont pas utilisés dans le calcul des coïncidences, car produits dans les temps morts du dispositif de comptage.

Les séquences temporelles dans chaque photomultiplicateur sont obtenues suivant la procédure suivante :

- le nombre de photons émis suite au dépôt monoénergétique et l'instant d'émission associé à chacun des

\section{Tableau 1}

Mise en évidence de la dépendance stochastique pour les coïncidences doubles entre deux photomultiplicateurs lorsque le temps de résolution décroît.

\begin{tabular}{|c|c|c|c|}
\hline $\begin{array}{c}\text { Temps de } \\
\text { résolution } \\
\text { (ns) }\end{array}$ & $P_{E}\left(x_{1} \cap x_{2}\right)$ & $P_{E}\left(x_{1}\right) \cdot P_{E}\left(x_{2}\right)$ & $\begin{array}{c}\text { Écart } \\
\text { relatif } \\
(\%)\end{array}$ \\
\hline 8 & $0,2627(2)$ & $0,2884(2)$ & 8,9 \\
\hline 16 & $0,3367(2)$ & $0,3472(2)$ & 3,0 \\
\hline 24 & $0,3602(2)$ & $0,3663(2)$ & 1,7 \\
\hline 32 & $0,3691(3)$ & $0,3737(3)$ & 1,2 \\
\hline 40 & $0,3735(2)$ & $0,3774(2)$ & 1,0 \\
\hline 80 & $0,3827(1)$ & $0,3852(1)$ & 0,6 \\
\hline 160 & $0,3920(1)$ & $0,3933(1)$ & 0,3 \\
\hline 240 & $0,3961(1)$ & $0,3966(1)$ & 0,1 \\
\hline 320 & $0,3980(2)$ & $0,3981(2)$ & $<0,1$ \\
\hline
\end{tabular}

photons sont générés de façon aléatoire selon deux modes de désexcitation (généralement appelés rapide et retardée en fluorescence moléculaire). Pour chaque mode, le nombre de photons est calculé à partir d'une distribution de Poisson et un deuxième tirage selon une loi de décroissance exponentielle attribue une date d'émission à chaque photon ;

- comme dans le modèle statistique, les photons sont distribués de façon équiprobable entre les trois photomultiplicateurs selon une loi multinomiale de probabilité $1 / 3$;

- un tirage suivant une loi binomiale est finalement appliqué à chaque photon pour déterminer sa transformation en photoélectron (on utilise comme probabilité un rendement quantique égal à 0,25 ).

Les paramètres des distributions aléatoires relatives à l'émission des photons ont été estimés de façon à retrouver approximativement l'évolution du taux des coïncidences doubles en fonction du temps de résolution donnée dans la figure 4. Le comptage des coïncidences doubles est calculé à partir de la durée relative entre les deux premiers photoélectrons détectés dans deux photomultiplicateurs différents. Le nombre moyen de photons émis a été fixé à 12 afin d'obtenir un nombre moyen de photoélectrons détectés dans un photomultiplicateur égal à 1 . Un ajustement satisfaisant avec les écarts relatifs présentés en figure 4 est obtenu en prenant les valeurs suivantes pour les deux modes de désexcitation : pour la composante rapide, un nombre moyen de photons égal à 11 avec une durée de vie moyenne de 7 ns et pour la composante retardée, un nombre moyen de photons égal à 1 avec une durée de vie moyenne de 100 ns. Les calculs montrent que les photons sont en majorité émis suivant la composante rapide.

\subsection{Résultats et interprétation}

Afin de vérifier l'indépendance stochastique en fonction du temps de résolution, le tableau 1 compare les calculs du rendement de détection des coïncidences doubles entre deux photomultiplicateurs en utilisant d'une part la probabilité mutuelle $P_{E}\left(x_{1} \cap x_{2}\right)$, d'autre part le produit 
Tableau 2

Mise en évidence de la dépendance stochastique pour les coïncidences triples lorsque le temps de résolution décroît.

\begin{tabular}{|c|c|c|c|}
\hline $\begin{array}{c}\text { Temps de } \\
\text { résolution } \\
\text { (ns) }\end{array}$ & $P_{E}\left(x_{1} \cap x_{2} \cap x_{3}\right)$ & $P_{E}\left(x_{1}\right) \cdot P_{E}\left(x_{2}\right) \cdot P_{E}\left(x_{3}\right)$ & $\begin{array}{c}\text { Écart } \\
\text { relatif } \\
(\%)\end{array}$ \\
\hline 8 & $0,1269(1)$ & $0,1551(1)$ & 18,2 \\
\hline 16 & $0,1923(1)$ & $0,2045(1)$ & 6,0 \\
\hline 24 & $0,2151(2)$ & $0,2217(2)$ & 3,0 \\
\hline 32 & $0,2235(2)$ & $0,2284(2)$ & 2,1 \\
\hline 40 & $0,2277(2)$ & $0,2317(2)$ & 1,7 \\
\hline 80 & $0,2364(1)$ & $0,2390(1)$ & 1,1 \\
\hline 160 & $0,2452(1)$ & $0,2465(1)$ & 0,5 \\
\hline 240 & $0,2494(1)$ & $0,2499(1)$ & 0,2 \\
\hline 320 & $0,2510(1)$ & $0,2511(1)$ & $<0,1$ \\
\hline
\end{tabular}

des probabilités individuelles $P_{E}\left(x_{1}\right) \cdot P_{E}\left(x_{2}\right)$. On rappelle que la probabilité mutuelle correspond au comportement réel dans le système de mesure et que les probabilités individuelles sont utilisées dans le modèle RCTD statistique «classique ». De façon similaire, le tableau 2 compare les calculs du rendement des coïncidences triples en utilisant d'une part la probabilité mutuelle $P_{E}\left(x_{1} \cap x_{2} \cap x_{3}\right)$, d'autre part le produit des probabilités individuelles $P_{E}\left(x_{1}\right) \cdot P_{E}\left(x_{2}\right) \cdot P_{E}\left(x_{3}\right)$.

À l'examen des résultats, il apparaît clairement que l'indépendance stochastique entre les photomultiplicateurs n'est plus vérifiée lorsque la durée du temps de résolution diminue. En outre les écarts relatifs entre probabilités mutuelles et produit des probabilités individuelles sont plus élevés pour les coïncidences triples que pour les doubles. Cette variation différente entre les deux types de coïncidences biaise la relation donnée par le modèle statistique « classique » entre la valeur RCTD et le rendement de détection des coïncidences doubles. Cet effet est directement observable sur la figure 7 où sont représentés les rendements des coïncidences doubles en fonction de la valeur RCTD : ceux calculés à partir des probabilités mutuelles simulées et ceux calculés pour les mêmes valeurs $R C T D$ avec la relation (17) du modèle RCTD monoénergétique. Lorsque le temps de résolution décroît, les calculs indiquent une différence croissante entre ces rendements de détection. Pour chaque valeur RCTD, cette différence représente le biais qu'entraînerait l'application du modèle statistique «classique " pour le temps de résolution correspondant (qui correspond à une surestimation de la valeur de l'activité).

Ce résultat peut être rapproché de celui donné en figure 6 dans le cas de ${ }^{51} \mathrm{Cr}$ (spectre d'énergie discret). En effet les taux de coïncidences doubles obtenus par défocalisation sont donnés pour un temps de résolution fixé à 240 ns (assez long pour obtenir l'indépendance stochastique). Dans ce cas, l'évolution observée correspond à celle donnée par le modèle statistique «classique » et donc à celle obtenue dans la figure 7 pour les coïncidences doubles calculées à l'aide de ce modèle. De même l'évolution des coïncidences doubles mesurée par variation du temps de résolution dans la figure 6 correspond à celle simulée dans la figure 7 .

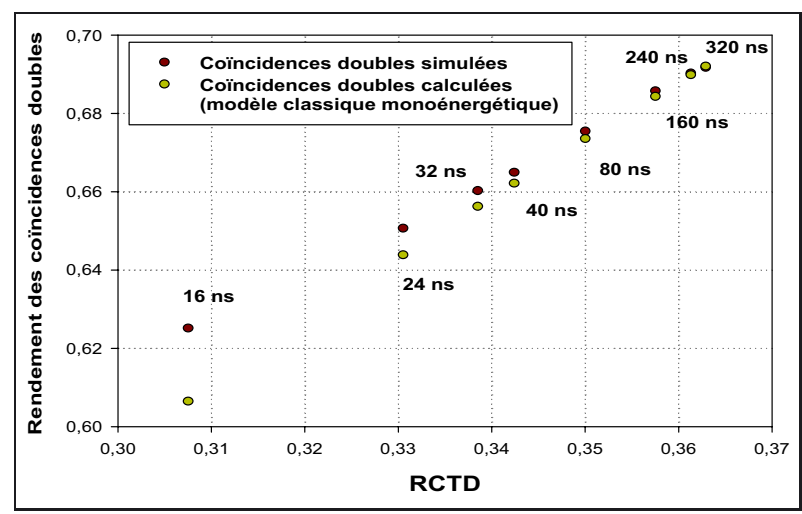

Fig. 7. - Comparaison entre les rendements de détection simulés et calculés à partir du modèle RCTD monoénergétique. Les écarts observés avec la réduction du temps de résolution sont corrélés avec l'existence d'une dépendance stochastique entre les photomultiplicateurs.

Parallèlement à la baisse des rendements de détection, les calculs montrent une décroissance du nombre moyen de photoélectrons détectés dans les photomultiplicateurs durant le temps de résolution lorsque celui-ci est réduit. On constate aussi une déviation entre ce nombre moyen et la variance lorsque l'indépendance stochastique n'est plus vérifiée. Comme attendu, le nombre moyen de photoélectrons par photomultiplicateur converge vers 1 pour les temps de résolution élevés; il est égal à 0,947 à $40 \mathrm{~ns}$ (avec une variance de 0,94 ) et il est égal à 0,736 à $8 \mathrm{~ns}$ (avec une variance de 0,68 ). Cette distorsion du processus poissonien a déjà été étudiée comme cause possible de biais dans le calcul de l'activité pour les émetteurs de basses énergies [13], sans pour autant donner une interprétation sur l'origine du phénomène [1]. Dans la présente étude, cette distorsion est interprétée comme la conséquence d'une dépendance stochastique entre les photomultiplicateurs.

\subsection{Commentaires}

De l'ensemble des résultats donnés par simulation, il est possible d'établir un lien entre la réduction du temps de résolution, l'existence d'une dépendance stochastique de type temporel entre les photomultiplicateurs et le risque d'un biais sur la détermination de l'activité. Lorsque le nombre de photons émis est faible, la condition d'indépendance stochastique doit être vérifiée pour obtenir l'adéquation entre le modèle RCTD statistique «classique » et le comportement réel du comptage des coïncidences. Comme exprimé dans la partie 2.2, ce problème est correctement décrit en utilisant les probabilités conditionnelles. Comme illustré sur le schéma de la figure 8 , la probabilité conditionnelle $P_{E}\left(x_{2} \mid x_{1}\right)$ n'est plus équivalente à la probabilité individuelle $P_{E}\left(x_{2}\right)$ lorsque le temps de résolution est trop court comparé à la distribution temporelle des photoélectrons. En effet, la probabilité de compter un photoélectron dans une voie est affectée par le déclenchement du temps de résolution, c'est-à-dire la première détection d'un photoélectron 


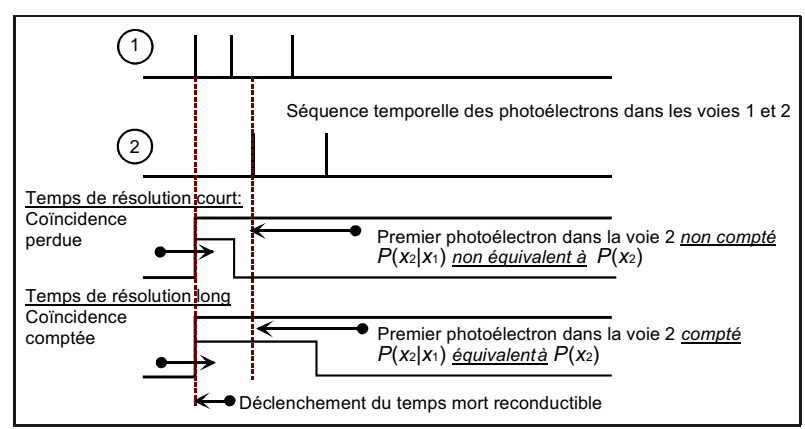

Fig. 8. - Influence du temps de résolution sur le comptage des coïncidences doubles et sur les probabilités conditionnelles.

dans une autre voie. Pour être compté, le deuxième photoélectron doit arriver avant la fin de ce temps de résolution. Cette condition introduit dans l'expression de la probabilité un terme qui dépend de la distribution temporelle des photoélectrons et de l'intervalle de temps séparant le dépôt d'énergie et l'arrivée du premier photoélectron. Cet effet est d'autant plus important que le nombre moyen de photoélectrons est faible ; a contrario il devient négligeable lorsque ce nombre est élevé (un calcul analytique détaillé pour un système à deux photomultiplicateurs est présenté par Bobin et coll. [5]).

En résumé, l'indépendance stochastique entre les photomultiplicateurs est une condition nécessaire à l'application du modèle RCTD statistique. Pour la mesure des émetteurs de basses énergies, elle est vérifiée lorsque le temps de résolution est adapté à la distribution temporelle de l'émission des photons relative à la décroissance de la fluorescence dans le scintillateur. Dans le cas particulier de la mesure de l'activité de ${ }^{3} \mathrm{H}$ dans le scintillateur Ultima Gold, un temps de résolution d'au moins 240 ns est suffisant pour le comptage des coïncidences.

\section{Application d'un nouveau modèle RCTD développé avec le code de simulation Monte-Carlo Geant4}

\section{1. Étude de l'émission Cherenkov consécutive à la diffusion Compton dans les faces d'entrée des photomultiplicateurs}

Le calcul de l'activité par la méthode RCTD nécessite la connaissance de l'énergie déposée dans le liquide scintillant. Pour un radionucléide émetteur $\gamma$, le dépôt d'énergie produit par diffusion Compton est calculé par simulation Monte-Carlo en considérant les interactions dans le volume du liquide scintillant Cependant le comptage de coïncidences observé en utilisant des sources sèches émettrices de photons $\gamma$, donc en l'absence de liquide scintillant, indique que l'émission lumineuse consécutive à la diffusion Compton ne peut se réduire au seul processus de scintillation dans le flacon. Les valeurs expérimentales obtenues pour différents radionucléides $\left({ }^{137} \mathrm{Cs}\right.$, ${ }^{54} \mathrm{Mn},{ }^{60} \mathrm{Co}$ ) sont données dans le tableau 3 ainsi que les énergies de l'émission $\gamma$ associée à chacun [12]. On remarque que les rapports $R C T D$ associés aux rendements

\section{Tableau 3}

Comparaison des valeurs calculées et expérimentales obtenues pour les rendements de coïncidences doubles et le rapport $R C T D$ pour des sources sèches émettrices de photons $\gamma$ en l'absence de liquide scintillant.

\begin{tabular}{|c|c|c|c|c|}
\hline \multirow{2}{*}{$\begin{array}{c}\text { Source } \\
(\mathbf{k e V})\end{array}$} & \multicolumn{2}{|c|}{$\begin{array}{c}\text { Rendement des } \\
\text { coïncidences doubles } \\
(\%)\end{array}$} & \multicolumn{2}{c|}{ RCTD } \\
\cline { 2 - 5 } & Mesure & Calcul & Mesure & Calcul \\
\hline $662\left({ }^{137} \mathrm{Cs}\right)$ & $0,66(5)$ & $0,69(4)$ & $0,15(1)$ & $0,16(1)$ \\
\hline $835\left({ }^{54} \mathrm{Mn}\right)$ & $1,00(4)$ & $1,2(1)$ & $0,16(1)$ & $0,17(1)$ \\
\hline $\begin{array}{c}1173-1332 \\
\left({ }^{60} \mathrm{Co}\right)\end{array}$ & $4,5(1)$ & $4,6(1)$ & $0,32(1)$ & $0,33(2)$ \\
\hline
\end{tabular}

des coïncidences doubles prennent des valeurs non négligeables. Par ailleurs, des mesures complémentaires réalisées par défocalisation des photomultiplicateurs donnent une réduction corrélée du rendement de détection des coïncidences doubles avec le rapport $R C T D$ comme pour une source radioactive mélangée dans un liquide scintillant. Les rendements de détection des coïncidences doubles donnés dans le tableau 3 indiquent aussi une diminution de ces valeurs lorsque l'énergie des photons $\gamma$ décroît, pouvant être interprétée comme un effet de seuil.

Compte tenu de ces résultats expérimentaux, l'hypothèse avancée est que l'émission lumineuse à l'origine du comptage de coïncidences doubles et triples serait sous forme de photons Cherenkov générés par les électrons produits par diffusion Compton dans les faces d'entrée des photomultiplicateurs. Ce phénomène a déjà été mentionné [14] sans pour autant être pris en considération dans la méthode RCTD.

La production de photons Cherenkov se manifeste lorsqu'une particule chargée se propage dans un milieu diélectrique transparent avec une vitesse supérieure à celle de la lumière dans ce milieu. L'interprétation théorique du phénomène a été donnée par Frank et Tamm [15] sur la base de l'électrodynamique classique à partir de laquelle il est possible d'établir les relations caractéristiques de l'effet Cherenkov. L'angle d'émission $\theta$ par rapport à la trajectoire de la particule chargée est défini en fonction de la vitesse $v$ et de l'indice de réfraction $n$ du milieu diélectrique selon l'expression suivante :

$$
\cos \theta=\frac{1}{\beta n} \quad \text { avec } \quad \beta=\frac{v}{c}
$$

où $c$ est la vitesse de la lumière dans le vide. L'angle d'émission tend vers un maximum lorsque $\beta$ tend vers 1.

Pour une longueur d'onde égale à 589 nm (raie jaune d'une lampe au sodium), l'indice de réfraction de la silice fondue est égal à 1,46 et l'angle maximum est d'environ $47^{\circ}$ dans la fenêtre d'entrée du photomultiplicateur XP2020Q. La condition d'apparition de l'effet Cherenkov est obtenue à partir de l'expression (18) :

$$
\beta \geq n^{-1}
$$


La théorie de la relativité donne l'expression de l'énergie de seuil d'apparition de l'effet Cherenkov pour un électron :

$$
E_{\mathrm{c}}=\left(\sqrt{\frac{1}{1-n^{-2}}}-1\right) \times 511 \mathrm{keV} .
$$

Ainsi le seuil Cherenkov est égal à $190 \mathrm{keV}$ dans la silice fondue pour une longueur d'onde de $589 \mathrm{~nm}(n=1,46)$. Une autre propriété importante est que la densité spectrale des photons Cherenkov est une fonction continue et décroissante selon le carré de la longueur d'onde; elle s'étend du domaine ultraviolet jusqu' au visible.

Dans le but de confirmer et d'évaluer cet effet, il a été fait appel à la simulation à l'aide du code Monte-Carlo Geant4 $[3,16]$. Ce code permet de calculer non seulement le transport des particules ionisantes mais aussi celui des photons optiques (y compris la création des photons Cherenkov). Il offre l'ensemble des fonctionnalités nécessaires à la modélisation de la géométrie de la cavité optique du système de détection spécifique à la méthode RCTD présenté dans la partie 3.1. Le modèle RCTD-Geant4 (détaillé dans § 5.2) a donc été développé pour simuler la production de photons Cherenkov suite à la diffusion Compton dans les faces d'entrée des photomultiplicateurs jusqu'à la création de photoélectrons conduisant au comptage des coïncidences. Dans ce but, le code de simulation Geant4 inclut la définition des propriétés optiques des différents éléments de la chambre optique pour gérer les processus de réflexion et de réfraction aux diverses interfaces. Cette caractéristique est nécessaire pour calculer les coïncidences obtenues entre les trois photomultiplicateurs lorsque l'émission des photons Cherenkov est produite dans la face d'entrée d'un seul photomultiplicateur. Le tableau 3 contient les résultats des calculs obtenus dans le cas de l'émission de photons $\gamma$ pour différents radionucléides : la cohérence avec les mesures expérimentales confirme sans ambiguïté l'hypothèse de l'émission de photons Cherenkov.

Le rendement lumineux de l'effet Cherenkov est inférieur à celui de la scintillation. En conséquence, l'émission de photons Cherenkov n' aura d'effet significatif que pour des radionucléides dont la mesure en scintillation liquide ne donne pas un haut rendement de détection. C'est par exemple le cas de ${ }^{54} \mathrm{Mn}$ pour lequel la valeur du rendement est de l'ordre de $50 \%$. Ce radionucléide se désintègre par capture électronique vers le niveau à $835 \mathrm{keV}$ de ${ }^{54} \mathrm{Cr}$ [12] conduisant à l'émission d'un photon $\gamma$ produisant des photons Cherenkov suite à la diffusion Compton dans les faces d'entrée des photomultiplicateurs. Les photons X et les électrons associés à la capture électronique ont des énergies essentiellement comprises entre $4 \mathrm{keV}$ et $6 \mathrm{keV}$ en grande partie absorbées dans le liquide scintillant.

Comme le montre la figure 9, la courbe du rendement de détection des coïncidences doubles en fonction du rapport $R C T D$ présente une valeur minimale de $R C T D$ au-dessus de laquelle deux valeurs du rendement peuvent être déduites pour un même rapport $R C T D$. Ce comportement est dû à la combinaison du spectre discret associé

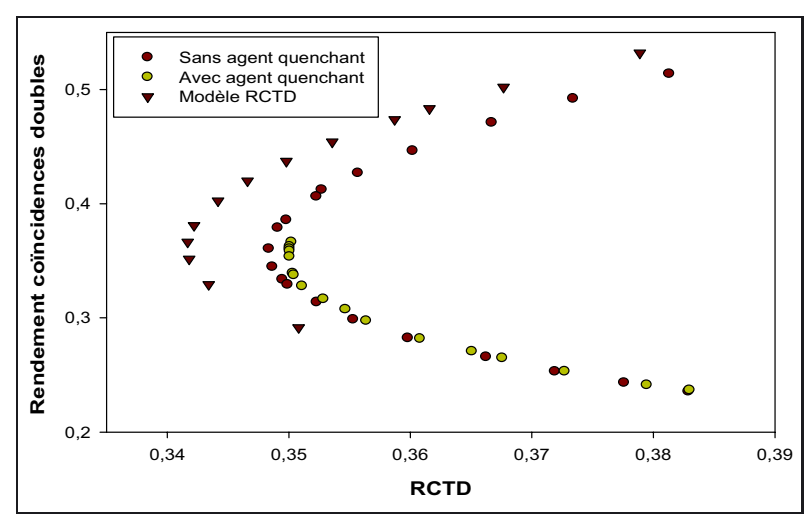

Fig. 9. - Comparaison de différentes relations entre le rendement de détection de coïncidences et le rapport RCTD pour ${ }^{54} \mathrm{Mn}$. Les points circulaires correspondent à des mesures expérimentales obtenues par défocalisation avec ou sans ajout d'agent quenchant. Les points triangulaires sont donnés par le modèle RCTD statistique sans prendre en compte l'émission Cherenkov dans les faces d'entrée des photomultiplicateurs.

à la capture électronique et du spectre continu de la diffusion Compton des photons $\gamma$ de $835 \mathrm{keV}$. La figure 9 donne deux séries de mesures obtenues par défocalisation pour une même source de ${ }^{54} \mathrm{Mn}$ d'activité connue dans le scintillateur commercial Ultima Gold. L'une d'elles a été réalisée après ajout d'un agent quenchant (nitrométhane) afin d'atténuer le rendement lumineux dans le flacon. La translation du point minimum RCTD atteste clairement de la non-équivalence entre les deux techniques utilisées pour réduire le rendement de détection. En effet, au niveau du point minimum $R C T D$, l'ajout d'agent quenchant affecte peu le rendement détection de l'émission Cherenkov contrairement à la technique de défocalisation qui a une incidence sur l'émission lumineuse provenant de l'ensemble de la cavité optique.

La figure 9 donne aussi les rendements de détection obtenus par calcul avec le modèle RCTD statistique «classique » [1] ne prenant pas en compte l'émission de photons Cherenkov dans les faces d'entrée des photomultiplicateurs. On constate à nouveau un déplacement de la courbe par rapport aux résultats expérimentaux qui conduira à un biais systématique dans le calcul de l'activité.

\subsection{Application du modèle RCTD-Geant4 à la méthode RCTD-Cherenkov : mesure d'activité du radiopharmaceutique ${ }^{11} \mathrm{C}$}

\subsubsection{Utilisation de l'émission Cherenkov comme alternative à la scintillation liquide}

La méthode RCTD peut être appliquée pour la mesure primaire d'activité en utilisant exclusivement l'effet Cherenkov. Bien que le rendement lumineux soit inférieur, cette alternative à la scintillation liquide offre quelques avantages intéressants. En effet, la mesure d'activité est directement applicable en solution aqueuse et ne nécessite donc pas de mélange avec un liquide scintillant. La préparation des sources radioactives est ainsi facilitée en évitant des risques d'instabilités chimiques comme 
ceux rencontrés pour la mesure de l'activité de ${ }^{11} \mathrm{C}$ : certaines réactions chimiques sont observables sous forme de chimiluminescence pouvant interférer avec la scintillation produite par le rayonnement ionisant. L'effet de seuil peut être aussi mis à profit pour discriminer le radionucléide à mesurer des impuretés possibles dans la solution radioactive. La mesure de ${ }^{32} \mathrm{P}\left(E_{\beta}^{-}\right.$max. d'environ $1710 \mathrm{keV}$ ) en est un bon exemple car, en général, les impuretés rencontrées sont sous forme d'émetteurs $\beta^{-}$tels que ${ }^{33} \mathrm{P}\left(E_{\beta}^{-}\right.$max. d'environ $\left.248 \mathrm{keV}\right)$ ou ${ }^{35} \mathrm{~S}$ ( $E_{\beta}^{-}$max. d'environ $167 \mathrm{keV}$ ) qui ont des énergies maximales inférieures au seuil Cherenkov dans une solution aqueuse (environ $260 \mathrm{keV}$ ).

L'extension du modèle RCTD statistique nécessite la prise en compte de l'anisotropie de l'émission des photons Cherenkov. Cette modification peut être réalisée en intégrant un paramètre libre supplémentaire [17]. Cet ajout peut être évité grâce à l'approche stochastique développée avec le code Geant4. En effet, le très bon accord des résultats de mesures et de calculs présentés dans le tableau 3 montre le bien-fondé de l'utilisation du code Geant4, notamment dans la prise en compte de l'effet Cherenkov. Ce constat a conduit le laboratoire à étendre le modèle stochastique RCTD-Geant4 d'abord à la méthode RCTD-Cherenkov, puis comme on le verra dans la partie 5.3, à la méthode RCTD par scintillation liquide.

Le modèle RCTD-Geant4 développé comme alternative au modèle classique pour l'émission Cherenkov a été validé avec la mesure d'activité de ${ }^{90} \mathrm{Y}$ [6]. Cette approche stochastique du modèle RCTD est décrite dans la suite dans le cas de la mesure primaire d'activité du radiopharmaceutique à durée de vie courte ${ }^{11} \mathrm{C}\left(T_{1 / 2}\right.$ de l'ordre de $20 \mathrm{~min}$ ).

\subsubsection{Description du modèle RCTD-Geant4 pour la mesure d'activité basée sur l'émission Cherenkov}

Dans le cas de la mesure d'activité basée sur l'émission Cherenkov, la simulation consiste à modéliser la production de photons Cherenkov sur le parcours de l'électron émis et leur transport jusqu'à la production de photoélectrons au niveau des photocathodes. Pour cela les propriétés optiques incluant les indices de réfraction sont attachées à chacun des éléments du modèle géométrique pour simuler les processus de réflexion et de réfraction aux interfaces [18]. La bande spectrale des photons simulés est limitée dans le domaine ultraviolet par la transmittance du flacon de verre (de l'ordre de $50 \%$ à $290 \mathrm{~nm}$ ) et par la réponse de la photocathode à basse fréquence (seuil de détection de l'ordre de $650 \mathrm{~nm}$ ).

La modélisation géométrique de la cavité optique est représentée en figure 10 : le flacon rempli avec une solution aqueuse, la cavité réflectrice en Téflon ${ }^{\circledR}$, les faces d'entrée des photomultiplicateurs en silice fondue. Les propriétés optiques définies pour chacun de ces éléments sont données dans la liste suivante :

- les indices de réfraction en fonction de la longueur d'onde de la solution aqueuse (volume de $15 \mathrm{~mL}$ ) sont

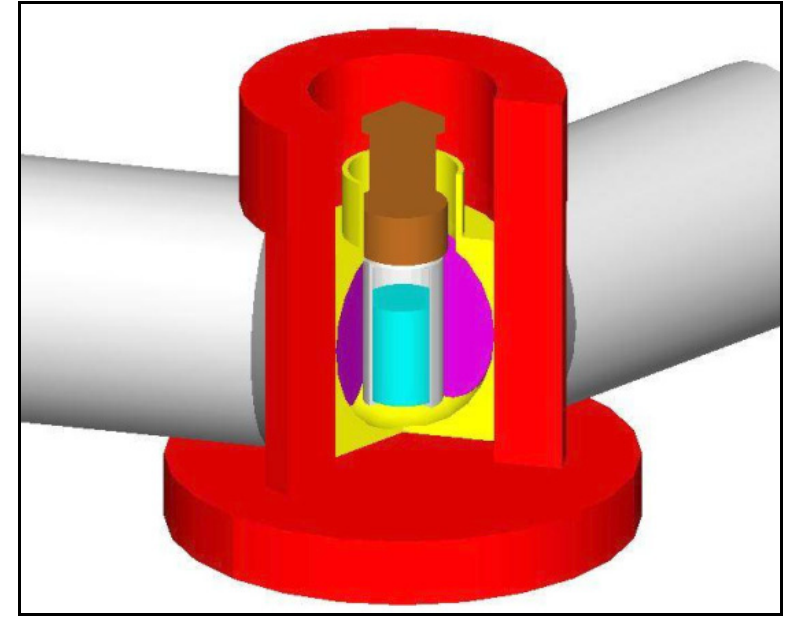

Fig. 10. - Modélisation géométrique du détecteur à trois photomultiplicateurs incluant le flacon au centre de la cavité réflectrice en Téflon ${ }^{\circledR}$.

définis en prenant ceux de l'eau. Le ménisque existant à la surface du liquide au bord du flacon est également modélisé ;

- les dimensions du flacon sont tirées de la littérature [19]; l'épaisseur de la paroi est de $1 \mathrm{~mm}$. Le verre borosilicate est modélisé comme un milieu dispersif (l'indice de réfraction à $400 \mathrm{~nm}$ est égal à 1,52). Les surfaces aux interfaces solution/verre et verre/air sont considérées comme polies ;

- la cavité réflectrice en Téflon ${ }^{\circledR}$ dans laquelle est intégré le flacon et où émergent les faces d'entrée des photomultiplicateurs est définie comme une surface réflectrice $(95 \%)$ et diffusante selon un profil de type lambertien ;

- la fenêtre d'entrée $(\varnothing=52 \mathrm{~mm})$ du photomultiplicateur en silice fondue est plane sur sa partie externe et sphérique en interne avec des épaisseurs comprises entre $1,5 \mathrm{~mm}$ et $7 \mathrm{~mm}$, du centre à la périphérie. La silice fondue est définie comme un milieu dispersif (l'indice de réfraction à $400 \mathrm{~nm}$ est égal à 1,47);

- la couche photoémissive $(\varnothing=46 \mathrm{~mm})$ de la photocathode bi-alkali $\left(\mathrm{K}_{2} \mathrm{CsSb}\right)$ est prise en compte grâce à ces propriétés optiques. L'interface silice fondue/photocathode est modélisée comme polie. La couche photoémissive est dispersive avec des indices de réfraction dépendant de la longueur d'onde tirés de la littérature [20,21] (l'indice de réfraction à $430 \mathrm{~nm}$ est égal à environ 2,5).

La simulation des évènements incluant le transport des positons émis par ${ }^{11} \mathrm{C}$ et des photons Cherenkov émis dans la cavité optique jusqu'à la production des photoélectrons dans les photocathodes, est implémentée suivant les étapes suivantes :

- les positons sont émis de façon aléatoire dans la solution aqueuse avec une distribution en énergie établie à partir des données nucléaires $\left(E_{\beta}^{+}\right.$max. 
d'environ $960 \mathrm{keV}$ ) [12] ; le code de calcul du spectre en énergie est tiré de la littérature [22];

- les photons sont créés sur le parcours du positon en respectant les caractéristiques propres à l'effet Cherenkov (effet de seuil, bande spectrale continue, émission selon un cône dont l'angle dépend de l'énergie du positon); le nombre de photons émis est calculé à partir d'une distribution poissonienne dont la valeur moyenne est calculée grâce à la théorie de Frank et Tamm [14];

- chaque photon émis est suivi dans son parcours dans la cavité optique en prenant en compte les processus de réfraction et réflexion aux différentes interfaces ;

- les photoélectrons créés au niveau des photocathodes sont calculés à partir des photons réfractés à l'interface avec la silice fondue en utilisant un rendement quantique fonction de la longueur d'onde dont les valeurs expérimentales pour le photomultiplicateur XP2020Q ont été publiées [23] (autour de $24 \%$ dans la région $300 \mathrm{~nm}-400 \mathrm{~nm}$ ). Le tirage aléatoire est de type binomial ;

- Pour chaque positon émis, la simulation donne un nombre de photoélectrons par photomultiplicateur à partir desquels il est possible d'obtenir au moins un comptage. Ce calcul est effectué grâce à un second tirage binomial avec un paramètre de focalisation représentant la probabilité pour un photoélectron d'atteindre la première dynode. Un seul succès est nécessaire pour donner un comptage dans un photomultiplicateur. Une coïncidence double est obtenue avec au moins deux succès dans deux photomultiplicateurs différents.

\subsubsection{Mesure de l'activité de ${ }^{11} \mathrm{C}$}

Quatre sources ont été préparées dans des flacons en verre préalablement remplis avec $15 \mathrm{~mL}$ de solution d'entraîneur inactive ayant la même composition que la solution étalon $\left(1 \mu \mathrm{g} \cdot \mathrm{g}^{-1}\right.$ de carbone sous forme de $\mathrm{NaCO}_{3} \mathrm{di}$ lué dans $\mathrm{H}_{2} \mathrm{O}$ ). La variation du rendement de détection a été effectuée par défocalisation des photomultiplicateurs : les valeurs expérimentales du rapport $R C T D$ sont comprises entre 0,25 et 0,3 .

La relation entre le rendement des coïncidences doubles et le rapport $R C T D$ a été calculée selon deux distances des faces d'entrée avec le centre de la cavité optique $(16 \mathrm{~mm}$ et $17 \mathrm{~mm})$ et deux séries de valeurs expérimentales de l'indice de réfraction de la photocathode bi-alkali $[20,21]$ en fonction de la longueur d'onde. Cette procédure est appliquée pour estimer la variabilité des résultats due à l'influence de paramètres affectant le nombre de photons réfractés à l'interface silice fondue/photocathode bi-alkali, et quantifier ainsi la composante de l'incertitude associée à la modélisation. La figure 11 montre les activités massiques en fonction des valeurs RCTD expérimentales calculées pour trois combinaisons de ces paramètres : Ha17 (distance de $17 \mathrm{~mm}$ et indice de réfraction donné par Harmer et al. [20]), Ha16

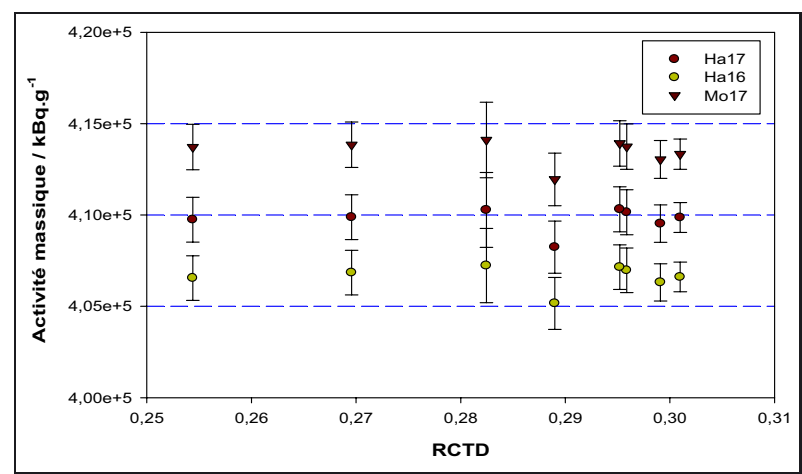

Fig. 11. - Activité massique de ${ }^{11} \mathrm{C}$ en fonction des valeurs expérimentales du rapport $R C T D$. Les trois séries de points sont obtenues par variation de paramètres ayant une influence sur le nombre de photons réfractés à la photocathode des photomultiplicateurs.

Tableau 4

Bilan d'incertitude associé à la mesure d'activité de ${ }^{11} \mathrm{C}$ par la technique RCTD-Cherenkov.

\begin{tabular}{|c|c|c|}
\hline Statistiques & $\begin{array}{l}\text { Distribution uniforme sur } \\
\text { les sources mesurées }\end{array}$ & $0,15 \%$ \\
\hline Mouvement propre & & $0,01 \%$ \\
\hline Temps actif & $\begin{array}{c}\text { Fréquence de l'horloge } \\
\text { à } 1 \mathrm{MHz}\end{array}$ & $0,1 \%$ \\
\hline Pesée & Méthode du pycnomètre & $0,05 \%$ \\
\hline $\begin{array}{c}\text { Modèle } \\
\text { RCTD-Geant4 }\end{array}$ & $\begin{array}{l}\text { Estimation conservative par } \\
\text { variation de paramètres ayant } \\
\text { une influence sur le nombre } \\
\text { de photoélectrons }\end{array}$ & $0,8 \%$ \\
\hline $\begin{array}{c}\text { Asymétrie des } \\
\text { photomultiplicateurs }\end{array}$ & $\begin{array}{l}\text { Estimation obtenue à partir } \\
\text { du modèle RCTD-Geant4 }\end{array}$ & $0,2 \%$ \\
\hline $\begin{array}{c}\text { Schéma de } \\
\text { désintégration }\end{array}$ & & $0,1 \%$ \\
\hline \multicolumn{2}{|c|}{ Incertitude type composée relative } & $0,85 \%$ \\
\hline
\end{tabular}

(distance de $16 \mathrm{~mm}$ ) et Mo17 (indice de réfraction donné par Motta et Schönert [21]). On n'observe pas de tendance particulière entre les activités massiques et les valeurs $R C T D$; l'inverse aurait indiqué une inadéquation significative entre la simulation donnée avec le modèle RCTD-Geant4 et le dispositif expérimental.

Le rendement de détection maximum des coïncidences doubles est d'environ 23,5\% correspondant à un paramètre de focalisation égal à 0,97 . Cette dernière valeur est cohérente avec celle obtenue pour la mesure de ${ }^{90} \mathrm{Y}\left(E_{\beta}^{-}\right.$max. d'environ $\left.2280 \mathrm{keV}\right)$ pour laquelle le rendement maximum obtenu en flacon verre était de environ $70 \%$. L'activité massique de la solution étalon de ${ }^{11} \mathrm{C}$ a été estimée à $410,0(35) \mathrm{MBq}^{-1} \mathrm{~g}^{-1}$ à la date de référence. Le bilan d'incertitude est donné dans le tableau 4. La composante d'incertitude associée à la décroissance radioactive n'est pas incluse pour la comparaison avec la valeur d'activité donnée par la méthode des coïncidences $4 \pi \beta-\gamma$.

L'activité massique de la solution étalon de ${ }^{11} \mathrm{C}$ a aussi été déterminée de façon indépendante avec la méthode des coïncidences $4 \pi \beta-\gamma$ [9]. Cette mesure a été 
effectuée avec une instrumentation classique : un compteur proportionnel (contenant du méthane à la pression atmosphérique) dans la voie de détection appelée voie $\beta$ (sensible aux positons) combiné à un détecteur à scintillation $\mathrm{NaI}(\mathrm{Tl})$ dans la voie $\gamma$ dédiée au comptage des photons $\gamma$ produits par annihilation électron-positon. Le dispositif électronique de comptage est basé sur une installation de mesure en anti-coïncidence développée au laboratoire [24]. Les sources mesurées sont sous forme de résidus secs obtenus après séchage d'une aliquote de la solution étalon déposée sur un support pouvant être introduit dans le compteur proportionnel. Le rendement de détection a été estimé à environ $98 \%$ dans le compteur proportionnel à partir du comptage des coïncidences. L'activité massique obtenue avec la méthode des coïncidences est de 411(2) $\mathrm{MBq}^{-1} \mathrm{~g}^{-1}$ à la date de référence. Cette valeur est compatible avec celle donnée par la méthode RCTD-Cherenkov avec le modèle RCTD-Geant 4.

Des résultats satisfaisants ont également été obtenus dans le cas de mesure d'activité de ${ }^{32} \mathrm{P}$ avec un rendement de détection des coïncidences doubles maximum de $57 \%$ $\left(E_{\beta}^{-}\right.$max. d'environ $1710 \mathrm{keV}$ ).

\subsection{Application du modèle RCTD-Geant4 à la scintillation liquide}

\subsubsection{Mesure du rendement de scintillation dans le scintillateur commercial Ultima Gold}

Le développement de la technique RCTD-Cherenkov peut être considéré comme une étape de validation du modèle RCTD-Geant4 pour la mesure primaire d'activité. L'extension du modèle RCTD-Geant4 à la scintillation liquide nécessite l'estimation du rendement de scintillation car le nombre de photons ne peut être calculé comme cela est possible pour l'effet Cherenkov avec la théorie de Frank et Tamm. On notera que dans le cas du modèle classique, cette valeur est intégrée dans le paramètre libre dont la variation sert au calcul de la relation entre le rendement de détection des coïncidences doubles et le rapport $R C T D$.

L'extension du modèle RCTD-Geant4 à la scintillation liquide est implémentée en remplissant le flacon avec $10 \mathrm{~mL}$ du scintillateur commercial Ultima Gold dont la composition atomique a été publiée par Broda et coll. [1]. Concernant la modélisation optique, l'indice de réfraction est pris égal à 1,5 pour une longueur d'onde d'émission moyenne de $430 \mathrm{~nm}$ (correspondant au bisMSB utilisé comme second fluorophore dans le mélange scintillant). Comme pour le modèle classique, la nonlinéarité de l'émission lumineuse due au quenching d'ionisation est calculée grâce à la formule semi-empirique de Birks donnée par l'expression (12) en utilisant un facteur $k B$ égal $0,01 \mathrm{~cm} \cdot \mathrm{MeV}^{-1}$. Contrairement à l'effet Cherenkov, les photons sont distribués de façon isotrope le long du trajet de l'électron. Le nombre de photons émis suit une distribution poissonienne.

La mesure du rendement de scintillation dans le scintillateur Ultima Gold a été possible en appliquant la méthode des coïncidences $4 \pi(\mathrm{SL}) \beta-\gamma$ [25] en combinant le détecteur à scintillation liquide $(\mathrm{SL})$ en voie $\beta$ avec un détecteur semi-conducteur HPGe en voie $\gamma$. Cette méthode permet de mesurer expérimentalement le rendement des coïncidences doubles sans avoir recours à un calcul utilisant la valeur du rapport $R C T D$. Le système de comptage fondé sur la technique des anticoïncidences [24] est identique à celui utilisé pour la mesure d'activité de ${ }^{11} \mathrm{C}$. Les deux radionucléides ${ }^{60} \mathrm{Co}$ et ${ }^{54} \mathrm{Mn}$ [12] ont été choisis pour la mesure du rendement de scintillation en raison de la différence des énergies déposées dans le liquide scintillant suite à une désintégration. Le rendement des coïncidences doubles est mesuré à partir du rapport $N_{\mathrm{c}} / N_{\gamma}$ où $N_{\mathrm{c}}$ et $N_{\gamma}$ sont respectivement les taux de comptage des coïncidences bêta-gamma et des photons $\gamma$. Une relation avec le rendement de scintillation est ensuite calculée grâce au modèle RCTD-Geant4. Ce calcul est effectué en fixant le paramètre de focalisation à 0,97 sur la base de l'expérience acquise avec les mesures d'activité par la technique RCTD-Cherenkov.

Le radionucléide ${ }^{60} \mathrm{Co}$ se désintègre par émission $\beta^{-}$ vers les niveaux excités de ${ }^{60} \mathrm{Ni}$ [12]. La désexcitation conduit principalement à l'émission de deux photons $\gamma$ en cascade $(1173 \mathrm{keV}$ et $1332 \mathrm{keV})$. Le rendement de détection maximum des coïncidences doubles déduit des mesures $4 \pi(\mathrm{SL}) \beta-\gamma$ est élevé : 0,97. Concernant les calculs avec le modèle RCTD-Geant4, on considère que ce rendement de détection est principalement le résultat du dépôt d'énergie donné par les électrons suite à la désintégration $\beta^{-}\left(E_{\beta}^{-}\right.$max. d'environ $\left.317 \mathrm{keV}\right)$. Le rendement de scintillation calculé à partir des mesures de ${ }^{60}$ Co donne une valeur comprise entre 7 et 8 photons par kiloélectronvolt.

Le radionucléide ${ }^{54} \mathrm{Mn}$ se désintègre par capture électronique vers le niveau excité d'environ $835 \mathrm{keV}$ de ${ }^{54} \mathrm{Cr}$ [12]. La désexcitation produit l'émission d'un photon $\gamma$ monoénergétique. Le rendement de détection des coïncidences doubles est déterminé en utilisant le rapport mesuré $N_{\mathrm{c}} / N_{\gamma}$ en centrant le comptage des photons $\gamma$ sur le pic d'absorption totale à $835 \mathrm{keV}$; une valeur égale à environ 0,54 est obtenue. Le calcul du rendement de détection avec le modèle RCTD-Geant4 est implémenté en considérant le dépôt d'énergie donné par la capture électronique. L'émission des photons $\mathrm{X}$ et des électrons Auger (énergies maximales comprises entre $4 \mathrm{keV}$ et $6 \mathrm{keV}$ ) suite au réarrangement du cortège électronique, est implémentée avec le modèle KLM dont la description a été publiée par Broda et coll. [1]. Le rendement de scintillation de Ultima Gold estimé à partir des mesures $4 \pi(\mathrm{SL}) \beta-\gamma$ prend des valeurs comprises entre 8 et 8,5 photons par kiloélectronvolt.

En raison de la non-linéarité de l'émission de scintillation suite à un dépôt de basse énergie, le rendement de détection des coïncidences ${ }^{54} \mathrm{Mn}$ calculé avec le modèle RCTD-Geant4 est plus sensible à la valeur du rendement de scintillation. En conséquence, une valeur égale à 8,2(3) photons par kiloélectronvolt a été adoptée; l'incertitude associée reflète la sensibilité à la variabilité du facteur $\mathrm{kB}\left(0,008 \mathrm{~cm} \cdot \mathrm{MeV}^{-1}-0,012 \mathrm{~cm} \cdot \mathrm{MeV}^{-1}\right)$. Cette 
Tableau 5

Résultats des simulations avec le modèle RCTD-Geant4 pour des dépôts de $5 \mathrm{keV}$ dans l'ensemble du liquide scintillant et selon l'axe central du flacon.

\begin{tabular}{|c|c|c|}
\hline & $\begin{array}{c}\text { Volume } \\
\text { SL }\end{array}$ & $\begin{array}{c}\text { Axe } \\
\text { central }\end{array}$ \\
\hline $\begin{array}{c}\text { RCTD simulé (TDCR-Geant4) } \\
\text { Rendement des coïncidences doubles } \\
\text { simulé }\end{array}$ & $0,3273(4)$ & $0,4076(5)$ \\
\hline $\begin{array}{c}\text { Rendement des coïncidences doubles } \\
\text { calculé }\end{array}$ & $0,6094(6)$ & $0,7492(6)$ \\
\hline Écart relatif & $0,6386(8)$ & $0,7450(9)$ \\
\hline$P_{E}\left(x_{1} \cap x_{2}\right)$ & $4,8 \%$ & $\approx 0,1 \%$ \\
\hline$P_{E}\left(x_{1}\right) \cdot P_{E}\left(x_{2}\right)$ & $0,3364(4)$ & $0,4530(5)$ \\
\hline$P_{E}\left(x_{1} \cap x_{2} \cap x_{3}\right)$ & $0,3315(4)$ & $0,4530(5)$ \\
\hline$P_{E}\left(x_{1}\right) \cdot P_{E}\left(x_{2}\right) \cdot P_{E}\left(x_{3}\right)$ & $1,5 \%$ & $/$ \\
\hline$\left.x_{2}\left(x_{1}\right) / P_{E}\left(x_{1}\right) \cdot P_{E}\left(x_{2}\right) \cdot P_{E}\left(x_{3}\right)\right]$ & $0,1995(3)$ & $0,3054(5)$ \\
\hline $1-\left[P_{E}\left(x_{1} \cap x_{2} \cap x_{2}\right)\right]$ & $0,1908(3)$ & $0,3050(5)$ \\
\hline Nombre moyen de photoélectrons & $4,6 \%$ & $\approx 0,1 \%$ \\
\hline Variance & $\approx 0,884$ & $\approx 1,19$ \\
\hline Écart relatif & $\approx 12 \%$ & $\approx 1 \%$ \\
\hline
\end{tabular}

estimation du rendement de scintillation obtenue à partir des mesures $4 \pi(\mathrm{SL}) \beta-\gamma$ est cohérente avec des valeurs antérieures données publiées par Fuchs et Laustriat [26].

\subsection{2. $\quad$ Étude de la dépendance stochastique due à la distribution des photons entre les photomultiplicateurs}

Les calculs avec le modèle RCTD-Geant4 [5] ont été effectués pour des dépôts d'électrons monoénergétiques ( $5 \mathrm{keV}, 8 \mathrm{keV}, 12 \mathrm{keV}$ et $20 \mathrm{keV}$ ) d'une part dans la totalité du volume du liquide scintillant, d'autre part en réduisant l'origine de l'émission à l'axe central du flacon dans le but d'approcher une distribution isotrope des photons entre les photomultiplicateurs. Les simulations ont été implémentées en utilisant un paramètre de focalisation fixé à 0,97 et un rendement de scintillation égal à 8,2 photons par kiloélectronvolt $\left(k B=0,01 \mathrm{~cm} \cdot \mathrm{MeV}^{-1}\right)$.

Dans le modèle RCTD statistique, le calcul des probabilités de détection des coïncidences est fondé sur l'isotropie de la distribution des photons entre les photomultiplicateurs suite à une désintégration. Dans l'approche classique, la position d'origine de l'émission lumineuse dans le liquide scintillant ainsi que les différents processus de réflexion et de réfraction dans la cavité optique ne sont pas considérés dans le calcul des probabilités. En suivant la même démarche que pour l'étude de l'influence du temps de résolution, les probabilités mutuelles de détection dans deux et trois voies de comptage sont comparées aux produits des probabilités individuelles correspondants grâce au modèle RCTD-Geant4. L'impact sur le rendement de coïncidences doubles est aussi étudié en utilisant l'expression analytique (17) propre au modèle RCTD statistique pour un dépôt monoénergétique.
Les tableaux 5 et 6 montrent les résultats correspondant aux dépôts monoénergétiques de $5 \mathrm{keV}$ et $20 \mathrm{keV}$. Dans les deux cas, on donne la valeur $R C T D$ et le rendement de détection des coïncidences doubles simulé avec le modèle RCTD-Geant4 (correspondant à un calcul avec les probabilités mutuelles). Le rendement de détection des coïncidences doubles donné par le modèle statistique pour un dépôt monoénergétique (17) est obtenu en prenant la valeur $R C T D$ simulée. Dans le cas du dépôt de $5 \mathrm{keV}$ dans le volume du liquide scintillant, un écart relatif de 4,8\% entre les rendements de détection simulé et calculé est obtenu; cet écart diminue pour les dépôts de $8 \mathrm{keV}$ (environ $2 \%$ ) et $12 \mathrm{keV}$ (environ 0,7\%) pour devenir négligeable pour un électron de $20 \mathrm{keV}$. En outre, ces écarts sont corrélés à des différences significatives entre les probabilités mutuelles et les produits des probabilités individuelles. Ces résultats indiquent que l'indépendance stochastique n'est pas vérifiée pour des dépôts de basses énergies ( $5 \mathrm{keV}, 8 \mathrm{keV}$ et $12 \mathrm{keV})$. La conséquence est un écart sur le calcul du rendement de détection des coïncidences doubles avec le modèle RCTD statistique.

Un écart significatif est également mis en évidence entre la valeur moyenne et la variance du nombre de photoélectrons évalués au moyen du modèle RCTD-Geant4. Ce résultat est en contradiction avec l'hypothèse poissonienne du modèle RCTD statistique. Bien que cette hypothèse ne soit pas vérifiée dans le cas d'un dépôt de $20 \mathrm{keV}$, l'indépendance stochastique est validée en raison du grand nombre de photons émis; en effet le rendement des coïncidences doubles est dans ce cas proche de 1 .

Les simulations effectuées pour un dépôt d'énergie réduit à l'axe central du flacon montrent des résultats totalement différents. En effet, les écarts constatés précédemment sont négligeables voire inexistants quelles que 
Tableau 6

Résultats des simulations avec le modèle RCTD-Geant4 pour des dépôts de $20 \mathrm{keV}$ dans l'ensemble du liquide scintillant et selon l'axe central du flacon.

\begin{tabular}{|c|c|c|}
\hline & $\begin{array}{c}\text { Volume } \\
\text { SL }\end{array}$ & $\begin{array}{c}\text { Axe } \\
\text { central }\end{array}$ \\
\hline $\begin{array}{c}\text { RCTD simulé (TDCR-Geant4) } \\
\text { simulé }\end{array}$ & $0,9491(7)$ & $0,9932(7)$ \\
\hline $\begin{array}{c}\text { Rendement des coïncidences doubles } \\
\text { calculé }\end{array}$ & $0,9985(7)$ & 1 \\
\hline $\begin{array}{c}\text { Écart relatif } \\
P_{E}\left(x_{1} \cap x_{2}\right)\end{array}$ & $0,9990(7)$ & 1 \\
\hline$P_{E}\left(x_{1}\right) \cdot P_{E}\left(x_{2}\right)$ & $<0,1 \%$ & $/$ \\
\hline$P_{E}\left(x_{1} \cap x_{2} \cap x_{3}\right)$ & $0,9644(7)$ & $0,9954(7)$ \\
\hline$P_{E}\left(x_{1}\right) \cdot P_{E}\left(x_{2}\right) \cdot P_{E}\left(x_{3}\right)$ & $0,9642(7)$ & $0,9954(7)$ \\
\hline$\left.P_{E}\left(x_{1} \cap x_{2}\right) / P_{E}\left(x_{1}\right) \cdot P_{E}\left(x_{2}\right)\right]$ & $<0,1 \%$ & $/$ \\
\hline $1-\left[P_{E}\left(x_{1} \cap x_{2} \cap x_{3}\right) / P_{E}\left(x_{1}\right) \cdot P_{E}\left(x_{2}\right) \cdot P_{E}\left(x_{3}\right)\right]$ & $0,9476(7)$ & $0,9931(7)$ \\
\hline Nombre moyen de photoélectrons & $0,9468(7)$ & $0,9931(7)$ \\
\hline Variance & $<0,1 \%$ & $/$ \\
\hline Écart relatif & $\approx 4,8$ & $\approx 6,09$ \\
\hline
\end{tabular}

soient les énergies. Cette configuration de l'émission lumineuse approchant l'isotropie de la distribution des photons dans la cavité optique n'introduit pas de dépendance stochastique entre les photomultiplicateurs; elle permet bien de retrouver les hypothèses à la base du modèle RCTD statistique.

Les simulations montrent donc qu'il existe une dépendance stochastique de type géométrique entre les photomultiplicateurs qui dépend de la position d'origine de l'émission lumineuse dans le liquide scintillant. Ce phénomène, qui s'accentue avec la diminution de l'énergie des électrons, a une conséquence directe sur le comptage des coïncidences. Cette dépendance stochastique peut être interprétée comme le résultat d'une redistribution des photons de scintillation entre les photomultiplicateurs due à une combinaison entre la position d'origine de l'émission lumineuse dans le liquide scintillant et les différents processus de réfraction et de réflexion dans la cavité optique (en particulier à l'interface verre/air du flacon). Pour les dépôts d'énergies inférieures à $20 \mathrm{keV}$, le modèle RCTD-Geant4 permet d'intégrer la complexité des multiples configurations de la distribution des photons dans la cavité optique. Comme décrit dans la partie 2.1, l'intégration de cet effet dans le modèle RCTD statistique nécessiterait l'usage du formalisme des probabilités conditionnelles. La dépendance stochastique génère aussi une distorsion de la distribution poissonienne de la production des photoélectrons qui est une hypothèse importante du modèle statistique. La littérature présente des études qui tentent d'expliquer les écarts de mesures d'activités d'émetteurs de basses énergies tels que ${ }^{3} \mathrm{H}[1]$. D'autres distributions ont été testées (binomiale, Polya) [13]. Il est intéressant de noter que ces investigations supposent que la distorsion de la distribution poissonienne est due au faible nombre de photoélectrons détectés suite à une désintégration. Cependant on constate dans le tableau 5 que pour un dépôt de $5 \mathrm{keV}$ selon l'axe central du flacon, l'hypothèse poissonienne est vérifiée malgré le faible nombre moyen de photoélectrons détectés dans un photomultiplicateur (environ 1,2). En fait la présente étude montre que la distorsion de la distribution poissonienne et le biais sur la mesure d'activité avec le modèle RCTD statistique résultent tous deux de la dépendance stochastique entre les photomultiplicateurs pour des dépôts de basses énergies.

\subsection{Mesure d'activité d'une solution étalon de ${ }^{51} \mathrm{Cr}$}

\subsubsection{Mesure d'activité par la méthode $4 \pi \beta-\gamma$}

Le radionucléide ${ }^{51} \mathrm{Cr}$ se désintègre par capture électronique selon deux branchements : vers le niveau fondamental $(90,1 \%)$ ou le niveau excité à $320 \mathrm{keV} \mathrm{de}{ }^{51} \mathrm{~V}$ $(9,9 \%)$. La désexcitation produit l'émission d'un photon $\gamma$ [12]. La mesure d'activité de ce radionucléide émetteur de basses énergies (électrons Auger et de photons $\mathrm{X}$ d'énergies maximales comprises entre $4 \mathrm{keV}$ et $6 \mathrm{keV}$ ) peut être réalisée par la méthode des coïncidences $4 \pi(\mathrm{SL}) \beta-\gamma$ et par la méthode RCTD.

La méthode $4 \pi(\mathrm{SL}) \beta-\gamma$ [25] a été appliquée avec l'instrumentation de mesure en anti-coïncidence mentionnée dans la partie 5.3.1 [24]. Six sources radioactives ont été préparées par mélange de $10 \mathrm{~mL}$ du scintillateur liquide Ultima Gold et d'une aliquote de la solution étalon de ${ }^{51} \mathrm{Cr}$. Le rapport $N_{\mathrm{c}} / N_{\gamma}$ qui estime le rendement de détection dans la voie $\beta$, est calculé en prenant une fenêtre de comptage $\gamma$ centrée sur le pic d'absorption totale à $320 \mathrm{keV}$. L'activité est déterminée grâce à la technique d'extrapolation [27] en procédant à une série de mesures pour différents rendements de détection 
Tableau 7

Bilan d'incertitude associé à la mesure de l'activité de ${ }^{51} \mathrm{Cr}$ par la méthode des coïncidences $4 \pi \beta-\gamma$.

\begin{tabular}{|c|c|c|}
\hline Statistiques & $\begin{array}{l}\text { Distribution uniforme sur les } \\
\text { sources mesurées }\end{array}$ & $0,2 \%$ \\
\hline Mouvement propre & & $0,2 \%$ \\
\hline Temps actif & Fréquence de l'horloge à $1 \mathrm{MHz}$ & $0,01 \%$ \\
\hline Pesée & Méthode du pycnomètre & $0,1 \%$ \\
\hline $\begin{array}{c}\text { Technique } \\
\text { d'extrapolation }\end{array}$ & $\begin{array}{l}\text { Ajustement linéaire appliqué sur } \\
\qquad 17 \text { mesures }\end{array}$ & $0,35 \%$ \\
\hline $\begin{array}{c}\text { Décroissance } \\
\text { radioactive }\end{array}$ & $\begin{array}{c}T_{1 / 2}\left({ }^{51} \mathrm{Cr}\right)=27,703(3) \\
\text { jours [12] }\end{array}$ & $0,1 \%$ \\
\hline $\begin{array}{l}\text { Coïncidences } \\
\text { accidentelles }\end{array}$ & Estimation conservative & $0,1 \%$ \\
\hline $\begin{array}{c}\text { Schéma de } \\
\text { désintégration }\end{array}$ & $\begin{array}{c}\text { Correction des différences des } \\
\text { probabilités de capture } \\
\text { électronique } P_{\mathrm{K}}[28]\end{array}$ & $0,05 \%$ \\
\hline \multicolumn{2}{|c|}{ Incertitude type composée relative } & $0,48 \%$ \\
\hline
\end{tabular}

en voie $\beta$ (scintillation liquide). La variation est effectuée par défocalisation des photomultiplicateurs. Grâce à la méthode des moindres carrés, un ajustement linéaire avec une fonction affine de la forme $\left.N_{\beta}=F\left(1-N_{\mathrm{c}} / N_{\gamma}\right)\right)$ est appliqué à 17 valeurs expérimentales correspondant à des rapports $N_{\mathrm{c}} / N_{\gamma}$ compris entre 0,34 et 0,49 . De la mesure des six sources radioactives, l'activité de la solution étalon de ${ }^{51} \mathrm{Cr}$ a été estimée égale à 22,96(11) MBq/g à la date de référence. Le bilan d'incertitude est présenté dans le tableau 7. On remarque une correction de la valeur d'activité obtenue par la technique d'extrapolation due à la différence des valeurs de probabilités de capture électronique sur la couche K entre les deux branchements du schéma de désintégration.

\subsubsection{Mesure de l'activité de ${ }^{51}$ Cr avec le modèle RCTD-Geant4}

La méthode RCTD a été appliquée avec les mêmes sources. Un temps de résolution de $240 \mathrm{~ns}$ a été utilisé pour le comptage des coïncidences entre les photomultiplicateurs. La réduction du rendement de détection a été également effectuée par défocalisation des photomultiplicateurs. L'évolution du taux de comptage des coïncidences doubles en fonction du rapport RCTD (compris entre 0,26 et 0,31 ) est représentée en figure 6 .

Le modèle RCTD-Geant4 développé pour la scintillation liquide a été utilisé pour le calcul du rendement de détection des coïncidences doubles. Le dépôt d'énergie dans le liquide scintillant dû aux photons $X$ et aux électrons émis suite à la capture électronique est implémenté avec le modèle KLM [1] en utilisant les données du schéma de désintégration disponibles dans la littérature [12]. La procédure mise en œuvre pour établir la relation entre le rendement de détection des coïncidences doubles et le rapport $R C T D$ expérimental est identique à celle adoptée pour la mesure RCTD-Cherenkov (voir $\S 5.2 .3$ ). Les résultats des calculs de l'activité pour différents rapports $R C T D$ avec le modèle RCTD-Geant4

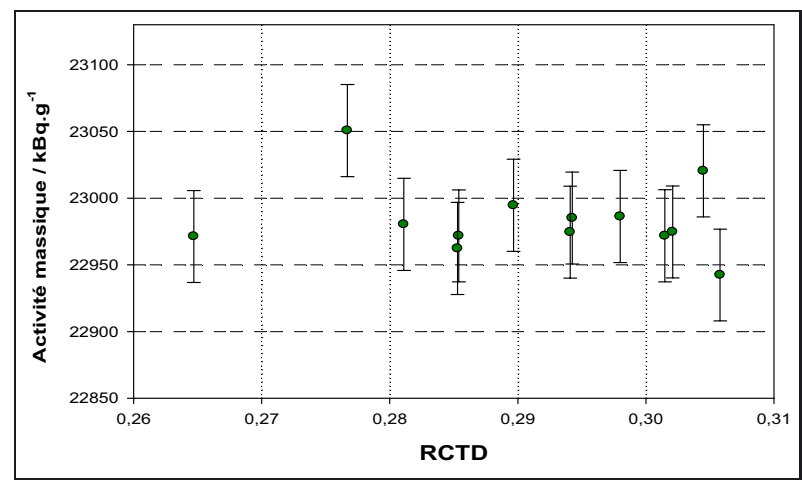

Fig. 12. - Activité massique de la solution étalon de ${ }^{51} \mathrm{Cr}$ calculée pour différents rapports RCTD expérimentaux avec le modèle RCTD-Geant4 (les incertitudes sont données avec un facteur d'élargissement $k=1$ ).

sont représentés dans la figure 12. Par exemple, pour un rapport $R C T D$ égal à 0,305 on obtient un rendement de détection des coïncidences doubles égal à $0,496(k B=$ $\left.0,01 \mathrm{~cm} \cdot \mathrm{MeV}^{-1}\right)$. L'activité massique de la solution étalon de ${ }^{51} \mathrm{Cr}$ avec le modèle RCTD-Geant4 a été estimée à 22,98(12) $\mathrm{MBq} \cdot \mathrm{g}^{-1}$ à la date de référence. Cette valeur est compatible avec celle donnée avec la méthode des coïncidences $4 \pi(\mathrm{SL}) \beta-\gamma$.

Le bilan d'incertitude est donné dans le tableau 8. La composante relative à la modélisation avec Geant4 est estimée par variation de la distance $(16 \mathrm{~mm}-17 \mathrm{~mm})$ des faces d'entrée des photomultiplicateurs avec le centre de la cavité optique. Cette procédure permet d'estimer l'influence du nombre de photoélectrons détectés au niveau des photocathodes sur le calcul du rendement de détection des coïncidences.

\subsubsection{Comparaison avec le modèle RCTD statistique}

La construction du modèle RCTD statistique est décrite dans la partie 2.2. Le calcul des dépôts d'énergie suite à une désintégration est similaire à celui utilisé pour le modèle RCTD-Geant4 : le dépôt d'énergie consécutif à la capture électronique est aussi implémenté avec le modèle KLM [1]. Les rendements de détection des photons $\mathrm{X}$ et le spectre en énergie du rayonnement $\gamma$ diffusé dans le liquide scintillant ont été calculés avec le code Geant4. La mesure d'activité avec le modèle RCTD sta-

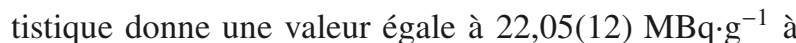
la date de référence : l'écart est de $4 \%$ avec celles données par la méthode des coïncidences $4 \pi(\mathrm{SL}) \beta-\gamma$ et avec le modèle RCTD-Geant4.

Cette sous-estimation de l'activité massique avec le modèle RCTD statistique est cohérente avec le résultat des simulations pour des dépôts monoénergétiques décrites dans la partie 5.3.2. On rappelle que pour une énergie de $5 \mathrm{keV}$, une différence de $4,8 \%$ est obtenue entre les rendements de détection des coïncidences doubles simulé et calculé. L'écart obtenu sur la mesure d'activité de ${ }^{51} \mathrm{Cr}$ avec le modèle RCTD statistique peut être interprété comme la conséquence d'une dépendance stochastique 
Tableau 8

Bilan d'incertitude associé à la mesure d'activité de ${ }^{51} \mathrm{Cr}$ obtenu avec le modèle RCTD-Geant 4 .

\begin{tabular}{|c|c|c|}
\hline Statistiques & $\begin{array}{c}\text { Distribution uniforme sur les } \\
\text { sources mesurées }\end{array}$ & $0,2 \%$ \\
\hline Mouvement propre & Fréquence de l'horloge à 1 MHz & $0,05 \%$ \\
\hline Temps actif & Méthode du pycnomètre & $0,01 \%$ \\
\hline Pesée & $\begin{array}{c}\text { Variation des paramètres ayant une } \\
\text { influence sur la production de } \\
\text { photoélectrons }\end{array}$ & $0,1 \%$ \\
\hline $\begin{array}{c}\text { Modèle } \\
\text { RCTD-Geant4 }\end{array}$ & $\begin{array}{c}T_{1 / 2}\left({ }^{51} \mathrm{Cr}\right)=27,703(3) \\
\text { jours }[12]\end{array}$ & $0,1 \%$ \\
\hline $\begin{array}{c}\text { Décroissance } \\
\text { radioactive }\end{array}$ & $\begin{array}{c}\text { Estimation conservative avec le } \\
\text { modèle RCTD-Geant4 }\end{array}$ & $0,2 \%$ \\
\hline $\begin{array}{c}\text { Asymétrie des } \\
\text { photomultiplicateurs }\end{array}$ & $\begin{array}{c}\text { Variation de la valeur entre } \\
\text { com.MeV }\end{array}$ & $0,1 \%$ \\
\hline \multicolumn{2}{|c|}{ et $0,008 \mathrm{~cm} \cdot \mathrm{MeV}^{-1}$} & $0,1 \%$ \\
\hline $\begin{array}{c}\text { Facteur } k B \\
\text { Schéma de } \\
\text { désintégration }\end{array}$ & Variation des valeurs $P_{\mathrm{K}}$ et $\omega_{\mathrm{K}}$ & $\mathbf{0 , 5 3 \%}$ \\
\hline \multicolumn{2}{|c|}{ Incertitude type composée relatif } \\
\hline
\end{tabular}

de type géométrique en raison du faible nombre de photons émis suite à une désintégration.

\subsection{Influence du flacon sur la dépendance stochastique de type géométrique entre photomultiplicateurs}

Il est intéressant de rapprocher ces observations d'une comparaison décrite dans la littérature [29] de mesures d'activité de ${ }^{55} \mathrm{Fe}$ effectuées avec différents types de flacons. Comme ${ }^{51} \mathrm{Cr}$, ${ }^{55} \mathrm{Fe}$ se désintègre par capture électronique et les énergies maximales émises sont comprises entre $5 \mathrm{keV}$ et $7 \mathrm{keV}$. Il ressort de cette étude qu'un écart de près de $3 \%$ a été obtenu sur la mesure d'activité de ${ }^{55} \mathrm{Fe}$ avec le modèle RCTD statistique «classique » entre des sources radioactives préparées dans des flacons en verre et en polyéthylène. La valeur la plus élevée étant obtenue avec les flacons en polyéthylène, cet effet peut être attribué à une réduction de la dépendance stochastique de type géométrique en raison des propriétés diffusives de ce matériau. En particulier, une modification des processus de réfraction et de réflexion des photons à l'interface flacon/air pourrait entraîner une distribution différente des photons dans la cavité optique entre les deux types de flacons.

Cette interprétation a été étayée en procédant à la même comparaison pour la mesure d'activité de ${ }^{90} \mathrm{Y}$ par la technique RCTD-Cherenkov. En raison de l'anisotropie de l'émission des photons Cherenkov, l'influence de la distribution des photoélectrons entre les photomultiplicateurs sur la relation entre le rendement des coïncidences doubles et le rapport $R C T D$ est bien mise en évidence par des mesures RCTD-Cherenkov. La figure 13 compare les taux de comptages des coïncidences doubles en fonction du rapport $R C T D$ pour les deux types de flacons (verre et polyéthylène) remplis avec une solution aqueuse de ${ }^{90} \mathrm{Y}$. La technique de défocalisation a été appliquée de façon identique dans les deux cas pour faire varier le rendement de détection. On observe une

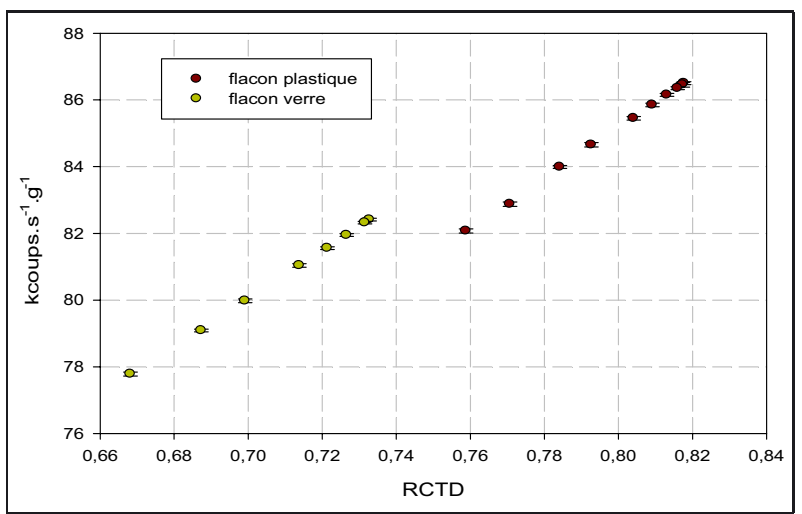

Fig. 13. - Comparaison de mesures RCTD-Cherenkov d'une solution aqueuse de ${ }^{90} \mathrm{Y}$ dans deux types de flacons (verre et polyéthylène). Les propriétés diffusives du flacon polyéthylène modifient la relation entre le rendement des coïncidences doubles et le rapport $R C T D$.

augmentation significative du rapport $R C T D$ dans le cas du flacon polyéthylène. Cet effet est conjugué à un déplacement de la relation entre le rendement des coïncidences doubles et le rapport $R C T D$. Comme pour l'étude avec ${ }^{55} \mathrm{Fe}$, cette différence entre les deux courbes peut être attribuée au caractère diffusif des parois du flacon polyéthylène qui modifie la distribution des photons entre les photomultiplicateurs.

\section{Discussions et perspectives}

Les études décrites dans cet article concernent en grande partie la mesure d'activité avec le modèle RCTD statistique «classique » de radionucléides émetteurs de basses énergies (inférieures à $20 \mathrm{keV}$ ) tels que ${ }^{3} \mathrm{H},{ }^{51} \mathrm{Cr}$, ${ }^{54} \mathrm{Mn}$. Pour cela, une nouvelle approche de la modélisation a été formalisée grâce aux probabilités conditionnelles afin d'interpréter des biais observés pour ce type de 
radionucléides comme conséquence d'une dépendance stochastique entre photomultiplicateurs. Bien que nécessaire à la construction du modèle RCTD statistique « classique », l'indépendance stochastique entre les photomultiplicateurs n'avait jamais été explicitement mentionnée dans les publications.

Le développement d'une nouvelle instrumentation pour la méthode RCTD basée sur la technologie numérique a permis d'étudier de façon systématique l'influence du temps de résolution sur le comptage des coïncidences entre les photomultiplicateurs. Une variation de l'activité mesurée en fonction du temps de résolution est observée en appliquant le modèle statistique «classique » pour les radionucléides émetteurs de basses énergies. Mis en évidence par des simulations temporelles, ce phénomène est la conséquence d'une dépendance stochastique entre les photomultiplicateurs en raison d'une perte systématique de coïncidences due à un temps de résolution trop court par rapport à la décroissance de la fluorescence. Cette étude permet de conclure qu'il faut un temps de résolution suffisamment long pour obtenir une bonne adéquation entre le modèle RCTD statistique «classique » et le comportement réel du système de détection. Dans le cas particulier de la mesure d'activité de ${ }^{3} \mathrm{H}$ dans le scintillateur Ultima Gold, il est ainsi préférable d'utiliser un temps de résolution d'au moins $240 \mathrm{~ns}$.

Une approche stochastique du modèle RCTD a été développée avec le code Monte-Carlo Geant4 afin d'intégrer les processus optiques qui ne sont pas pris en compte dans le modèle statistique : effet Cherenkov, processus de réfraction et de réflexion aux interfaces dans la cavité optique, propagation des photons jusqu'à la production des photoélectrons. Ce nouveau modèle RCTD-Geant4 a permis de confirmer l'hypothèse que des coïncidences pouvaient être comptées suite à la production de photons Cherenkov par les électrons générés par diffusion Compton dans les faces d'entrée des photomultiplicateurs.

Le modèle RCTD-Geant4 a également permis le développement d'une nouvelle technique de mesure primaire utilisant l'effet Cherenkov comme alternative à la scintillation liquide; un des avantages de la technique RCTD-Cherenkov est la mesure directe de l'activité en solution aqueuse. Cette technique a été validée pour différents radionucléides dont le radiopharmaceutique à durée de vie courte ${ }^{11} \mathrm{C}\left(T_{1 / 2} \sim 20 \mathrm{~min}\right)$. La technique RCTD-Cherenkov a été récemment retenue dans le cadre du projet européen MetroMRT (Metrology for Molecular Radiotherapy) pour le développement d'une référence primaire des microsphères marquées à l'yttrium-90 [30] (ce radionucléide est utilisé pour le traitement du cancer du foie par radioembolisation).

Grâce à l'intégration de la scintillation au modèle RCTD-Geant4, l'étude sur la dépendance stochastique a été étendue à l'influence de la position d'origine de l'émission lumineuse dans le flacon pour des dépôts de basses énergies. De façon similaire à la dépendance stochastique de type temporel, des écarts significatifs ont été obtenus sur le calcul des rendements de détection des coïncidences doubles avec le modèle RCTD statistique « classique ». Les simulations ont montré l'existence d'une dépendance stochastique de type géométrique due à une combinaison entre l'origine de l'émission lumineuse dans le flacon et les processus d'optique géométrique dans la cavité optique comme en particulier la réfraction et la réflexion à l'interface verre/air du flacon. L'adéquation entre le modèle statistique «classique » et la modélisation RCTD-Geant4 est retrouvée lorsque l'émission lumineuse est réduite à l'axe central du flacon; cette configuration correspond à une émission isotrope pour laquelle l'indépendance stochastique entre les photomultiplicateurs est vérifiée. Le problème de la dépendance stochastique de type géométrique a été mis en évidence expérimentalement pour la mesure d'activité de ${ }^{51} \mathrm{Cr}$ en utilisant le scintillateur Ultima Gold dans des flacons en verre. En effet des mesures d'activité cohérentes ont été obtenues entre le modèle RCTD-Geant4 et la méthode des coïncidences $4 \pi \beta-\gamma$. En revanche, une sousestimation de l'activité a été observée lorsque le modèle RCTD statistique «classique » était utilisé.

L'application du modèle RCTD-Geant4 pour la scintillation liquide n'a pas été limitée à la mesure d'activité d'émetteurs d'énergies inférieures à $20 \mathrm{keV}$. Des résultats satisfaisants ont été récemment obtenus pour la mesure de l'émetteur $\beta^{-63} \mathrm{Ni}\left(E_{\beta}^{-}\right.$max. d'environ $\left.67 \mathrm{keV}\right)$ [31]. Par ailleurs, l'ajout au modèle d'un détecteur sensible au rayonnement $\gamma$ permettra d'étendre son utilisation à la méthode des coïncidences $4 \pi(\mathrm{SL}) \beta-\gamma$. Ce nouvel outil sera particulièrement utile pour prédire les risques de biais de la mesure d'activité lors de l'application de la méthode d'extrapolation [27].

Au final, les études présentées dans cet article participent au travail métrologique visant à mieux connaître le domaine de validité du modèle RCTD statistique « classique », et le cas échéant, à mettre en évidence des solutions pour l'étendre.

\section{Remerciements}

Les auteurs tiennent particulièrement à remercier les personnes ayant participé à la préparation des sources radioactives pour les mesures d'activités : Didier Lacour, Isabelle Le Garrérès, Valérie Lourenço et Sophie Morelli.

\section{Références}

[1] Broda R., Cassette Ph. et Kossert K., "Radionuclide metrology using liquid scintillation", Metrologia, 44, 2007, S36-S52.

[2] Bobin Ch., Bouchard J. et Censier B., "First results in the development of an on-line digital counting platform dedicated to primary measurements", Appl. Radiat. Isot., 68, 2010, 1519-1522.

[3] Agostinelli S., Allison J., Amako K. et al., "Geant4 a simulation toolkit", Nucl. Instrum. Methods A, 506, 2003, 250-303. 
[4] Thiam C., Bobin Ch. et Bouchard J., "Simulation of Cherenkov photons emitted in photomultiplier windows induced by Compton diffusion using Monte Carlo code Geant4", Appl. Radiat. Isot., 68, 2010, 1515-1518.

[5] Bobin Ch., Thiam C., Chauvenet B. et Bouchard J., "On the stochastic dependence between photomultipliers in the TDCR method", Appl. Radiat. Isot., 70, 2012, 770780 .

[6] Bobin Ch., THIAM C. et Bouchard J., "Application of a stochastic TDCR model based on Geant 4 for Cherenkov primary measurements", Appl. Radiat. Isot., 68, 2010, 2366-2371.

[7] Thiam C., Bobin Ch. et Bouchard J., "Radiopharmaceutical ${ }^{11} \mathrm{C}$ activity measurements by means of the TDCR-Cherenkov method based on a Geant4 stochastic modeling", Proceedings of the 2010 International Liquid Scintillation Conference (LSC2010), 6-10 sept. 2010, 341-348.

[8] BIRKS J.B., "Theory of the response of organic scintillation crystals to short-range particles", Phys. Rev., 86, 1952, 569.

[9] CAMPION P.J., "The standardization of radioisotopes by the beta-gamma coincidence method using high efficiency detectors", Int. Journal of Appl. Radiat. Isot., 4, 1959, 1519-1522.

[10] Bouchard J. et Cassette Ph., "MAC3: an electronic module for the processing of pulses delivered by a three photomultiplier liquid scintillation counting", Appl. Radiat. Isot., 52, 2000, 669-672.

[11] Steele T., Mo L. Bignell L., Smith M. et Alexiev D., "FASEA: a FPGA acquisition system and software event analysis for liquid scintillation counting", Nucl. Instrum. Methods A, 609, 2009, 217-220.

[12] BÉ et al., "Table of Radionucleides", BIPM Monographie BIPM-5, édité par le BIPM, Pavillon de Breteuil, F-92 312 Sèvres Cedex.

[13] BRODA R. et JECZMIENIOWSKI A., "Statistics of the LSdetector in the case of low counting efficiency", Appl. Radiat. Isot., 60, 2004, 453-458.

[14] Ross H.H., "Measurement of $\beta$-emitting nuclides using Cerenkov radiation", Anal. Chem., 41, 10, 1969, 12601265.

[15] FRANK I.M. et TAMM I.G., "Coherent visible radiation of fast electrons passing through matter", Dokl. Akad. Nauk. SSSR, 14, 1937, 109-114.

[16] Allison J. et al., "Geant4 developments and applications", IEEE Trans. Nucl. Sci., 53, 2006, 270-549.

Article reçu le 25 septembre 2012, version révisée reçue le 24 septembre 2013.
[17] KosSERT K., "Activity standardization by means of a new TDCR-Cerenkov technique", Appl. Radiat. Isot., 68, 2010, 1116-1120.

[18] LEVIN A. et MoISAN C., "A more physical approach to model the surface treatment of scintillation counters and its implementation into DETECT", Records of the 1996 IEEE Nuclear Science Symposium, 2, 1996, 702-706.

[19] CASsETTE P. et al., "Comparison of calculated spectra for the interaction of photons in a liquid scintillator. Example of ${ }^{54} \mathrm{Mn} 835 \mathrm{keV}$ emission", Appl. Radiat. Isot., 64, 2006, 1471-1480.

[20] HARMER S.W., Downey R., WANG Y. et TOWSEND P.D., "Variation in optical constants between photocathodes", Nucl. Instrum. Methods A, 564, 2006, 439-450.

[21] MotTA D. et SHÖNERT S., "Optical properties of bialkali photocathodes", Nucl. Instrum. Methods A, 539, 2005, 217-235.

[22] WILKINSON D.H., "The evaluation of finite nuclear size effects in allowed $\beta$-decay", Nucl. Phys. A, 158, 1970, 476-486.

[23] ARAúJo H.M. et al., "Study of bialkali photocathodes below room temperature in UV/VUV region", IEEE Trans. Nucl. Sci., 45, 3, 1998, 542-549.

[24] BOUCHARD J., "A new set of electronic modules (NIM standard) for a coincidence system using pulse mixing method", Appl. Radiat. Isot., 56, 2002, 269-273.

[25] Bobin C., Bouchard J., Hamon C., Iroulart M.G. et PlAGNARD J., "Standardization of ${ }^{67} \mathrm{Ga}$ using a $4 \pi(\mathrm{LS}) \beta-\gamma$ anticoincidence system", Appl. Radiat. Isot., 65, 2007, 757-763.

[26] Fuchs C. et Laustriat G., « Mesure du rendement absolu de radioluminescence des scintillateurs liquides à base de benzène et de ses dérivés méthylés », Rev. Phys. Appl., 5, 1970, 617-623.

[27] BAERG A.P., "Absolute measurement of radioactivity", Metrologia, 3, 4, 1967, 105-108.

[28] FUnCK E. et NYlandsted LARSEN A., "The influence from low energy x-rays and Auger electrons on $4 \pi \beta-\gamma$ coincidence measurements of electron-capture-decaying nuclides", Appl. Radiat. Isot., 34, 1983, 565-569.

[29] Simpson B.R.S., VAN WYNGAARDT W.M. et LUBbE J., "Fe-55 activity measurements at the NMISA revisited", Appl. Radiat. Isot., 68, 2010, 1529-1533.

[30] DEZARn A.D. et al., "Recommendations of the American Association of Physicists in Medicine on dosimetry, imaging, and quality assurance procedures for ${ }^{90} \mathrm{Y}$ microsphere brachytherapy in the treatment of hepatic malignacies", Med. Phys., 38, 8, 2011, 4824-4844.

[31] Thiam C., Bobin Ch., Chauvenet B. et Bouchard J., "Application of TDCR-Geant4 modeling to standardization of ${ }^{63} \mathrm{Ni}$ ", Appl. Radiat. Isot., 70, 2012, 2195-2199. 